\title{
The physics of radioembolization
}

\author{
Remco Bastiaannet ${ }^{1 *}$ D, S. Cheenu Kappadath², Britt Kunnen ${ }^{1}$, Arthur J. A. T. Braat ${ }^{1}$, Marnix G. E. H. Lam
} and Hugo W. A. M. de Jong ${ }^{1}$

\author{
* Correspondence: \\ r.bastiaannet@umcutrecht.nl \\ ${ }^{1}$ Department of Radiology and \\ Nuclear Medicine, University \\ Medical Center Utrecht, Room \\ E01.132, P.O. Box 85500, 3508, GA, \\ Utrecht, The Netherlands \\ Full list of author information is \\ available at the end of the article
}

\begin{abstract}
Radioembolization is an established treatment for chemoresistant and unresectable liver cancers. Currently, treatment planning is often based on semi-empirical methods, which yield acceptable toxicity profiles and have enabled the largescale application in a palliative setting. However, recently, five large randomized controlled trials using resin microspheres failed to demonstrate a significant improvement in either progression-free survival or overall survival in both hepatocellular carcinoma and metastatic colorectal cancer. One reason for this might be that the activity prescription methods used in these studies are suboptimal for many patients.

In this review, the current dosimetric methods and their caveats are evaluated. Furthermore, the current state-of-the-art of image-guided dosimetry and advanced radiobiological modeling is reviewed from a physics' perspective. The current literature is explored for the observation of robust dose-response relationships followed by an overview of recent advancements in quantitative image reconstruction in relation to image-guided dosimetry.

This review is concluded with a discussion on areas where further research is necessary in order to arrive at a personalized treatment method that provides optimal tumor control and is clinically feasible.
\end{abstract}

Keywords: Radioembolization, Personalized medicine, Dosimetry, Radiobiological model, Dose-effect relationship, Theranostics

\section{Background}

Radioembolization is an established treatment for chemoresistant and unresectable liver cancers. The treatment consists of the administration of microspheres that are loaded with a beta-emitter into the arterial hepatic vasculature. As a result of a differential vasculature of the healthy liver and tumor tissue, the microspheres preferentially accumulate in the tumor tissue, resulting in a local radiation dose to the tumor whilst sparing healthy liver tissue.

Currently, two types of microspheres are approved for clinical use by the FDA and are CE-marked: resin microspheres (SIR-spheres; SirTex Medical) and glass microspheres (TheraSphere; BTG International Ltd.), both of which are loaded with ${ }^{90} \mathrm{Y}$. A third type consists of ${ }^{166} \mathrm{Ho}$-loaded poly-lactate spheres, called QuiremSpheres, which is yet to receive FDA approval but has been CE-marked.

Radioembolization treatment planning is currently based on semi-empirical methods, which are designed to yield acceptable toxicity profiles and have enabled the 
large-scale application in a palliative setting. The addition of radioembolization with SIR-spheres to first-line treatments for metastatic colorectal cancer was investigated in three large randomized controlled trials, SIRFLOX [1], FOXFIRE [2], and FOXFIRE-global. The combined analyses of these three trials did not show a significant improvement in either progression-free survival [3] or overall survival [4]. Similarly, the SARAH and SIRveNIB Phase III studies failed to show an improvement in overall or progression-free survival after the treatment of advanced hepatocellular carcinoma with SIR-spheres vs. sorafenib [5, 6].

One reason for this might be that the current activity planning methods often result in underdosing (and in some cases overdosing) in patients [1, 2, 7-9]. Fortunately, a recent survey amongst European institutes has shown that some form of absorbed dose-based prescription was used by 64 and $96 \%$ of the respondents for the use of resin and glass microspheres, respectively [10]. The lack of biological clearance of the microspheres simplifies dosimetry compared to most other molecular radiotherapies. In order to further increase the adoption of absorbed dose-based prescription, the package inserts of both manufacturers could be improved by placing more emphasis on this type of activity prescription. Furthermore, there is mounting evidence for clear dose-effect relationships (see Table 1). However, the estimated absorbed dose needed to elicit a reliable tumor response or complication varies between studies. As such, reliable absorbed dose targets and limits are yet to be established.

This review aims to investigate the current state-of-the-art of dosimetry in relation to this discussion from a physics perspective, elaborating on technical difficulties and providing an overview of the relevant hiatuses in the current knowledge.

\section{Review}

Current activity planning methods

Pre-treatment safety procedure

Before the infusion of the therapeutic dose, an angiographic work-up is performed in which the hepatic vessel anatomy is explored and an infusion site is selected. As per EANM guidelines, this is followed by the administration of $75-150 \mathrm{MBq}$ of the surrogate particle ${ }^{99 \mathrm{~m}}$ Tc macroaggregated albumin $\left({ }^{99 \mathrm{~m}} \mathrm{Tc}-\mathrm{MAA}\right)$ [11]. These imageable protein aggregations aim to simulate the expected distribution of the subsequent therapeutic microspheres. There are three main reasons for the use of a simulation procedure using ${ }^{99 \mathrm{~m}}$ Tc-MAA [12].

First, extrahepatic depositions can be detected. This used to be done using planar scintigraphy; however, SPECT/CT has been shown to be superior for this goal [13].

Second, the lung shunt fraction (LSF) is estimated. This fraction is used as a proxy for the absorbed lung dose and is subsequently used to adjust the prescribed activity, as described in the next sections. The microsphere manufacturers specify that this estimation should be performed on planar scintigraphic imaging [14], according to the formula

$$
\mathrm{LSF}=\frac{C_{\text {lungs }}}{C_{\text {lungs }}+C_{\text {liver }}},
$$

where $C_{\text {lungs }}$ indicates the total counts in the lungs, and $C_{\text {liver }}$ the total counts in the liver. Usually, the number of counts in these regions-of-interest (ROIs) is determined on the geometric mean of the anterior and posterior views. However, the validity 


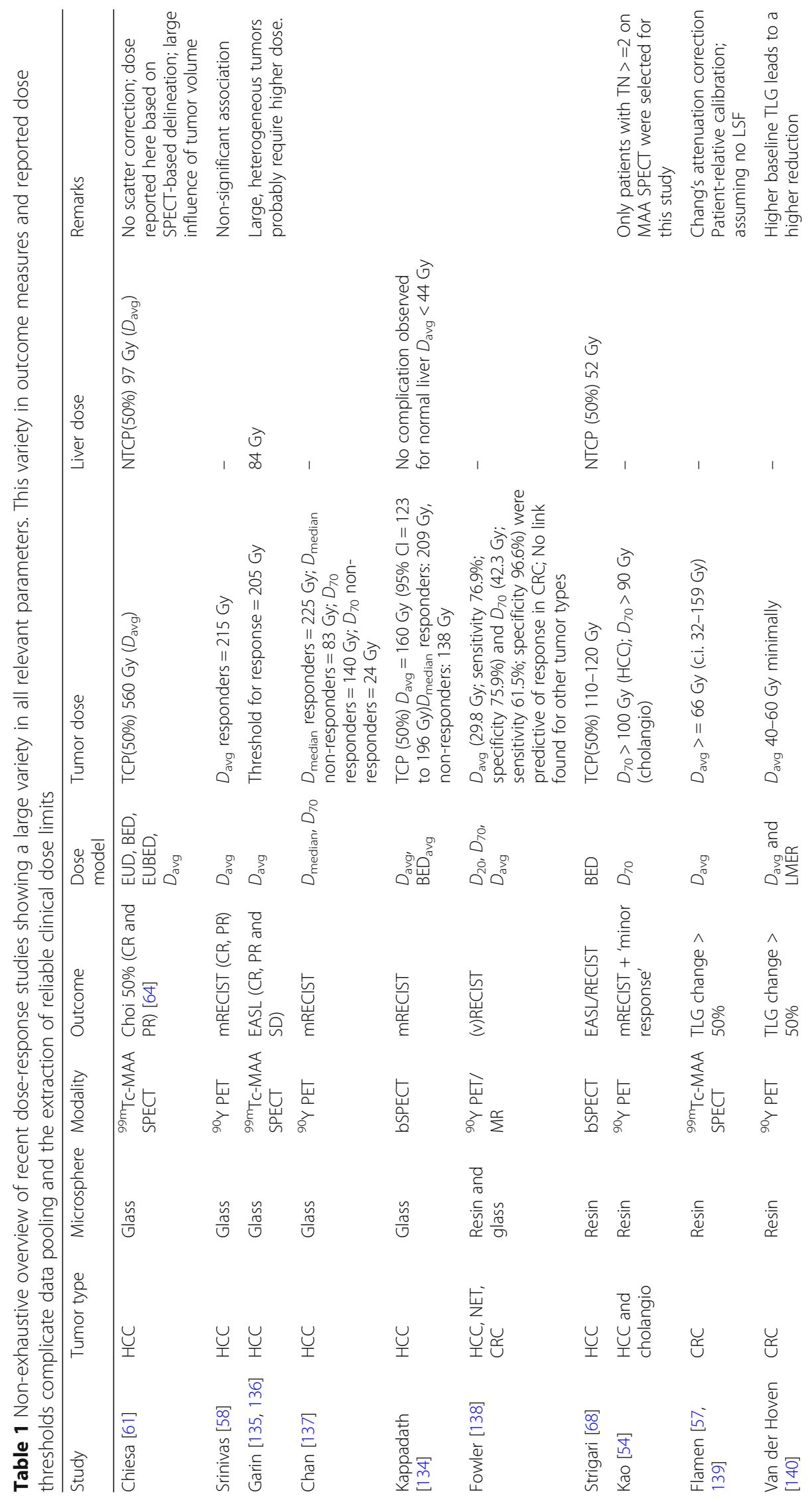




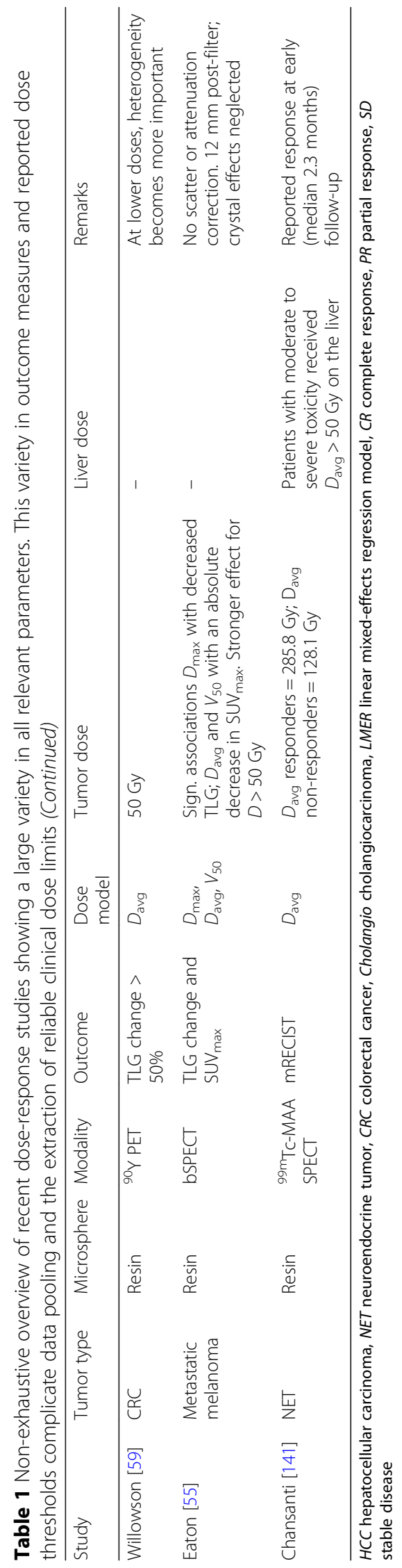


method has been questioned as it does not include proper compensation for differences in attenuation between the liver and the lungs, resulting in a systematic overestimation of LSF estimated on planar images relative to LSF estimated on SPECT/CT images [15-17].

Third, by using the ${ }^{99 \mathrm{~m}} \mathrm{Tc}-\mathrm{MAA}$ distribution as a predictor for the subsequent ${ }^{90} \mathrm{Y}$ distribution, it may be used for multi-compartment dosimetry (see the section "Multi-compartment dosimetry") [18].

The type of planning method used in clinical practice depends on the type of microsphere. For resin microspheres, the most commonly used method is the body surface area-based (BSA) method [14]. For glass microspheres and holmium-loaded microspheres, a commonly used method is the MIRD mono-compartment method [19-21]. Collectively, these methods are referred to as semi-empirical methods.

\section{BSA-based method for resin microspheres}

The BSA-based method was developed to overcome the clinically observed high toxicity of a previous method used in early clinical studies [22]. The prescribed activity using this previous method ranged between 2 and $3 \mathrm{GBq}$, depending on tumor load only and not on the liver size [14]. Conversely, the BSA-based method is based on the observation that BSA correlates with liver volume in the healthy population [23]. As such, the planned activity is adjusted to an individual patient's liver volume. The activity is calculated according to the following relationship [14]:

$$
A[\mathrm{GBq}]=\left(\mathrm{BSA}\left[m^{2}\right]-0.2\right)+\frac{V_{\text {tumor }}}{V_{\text {tumor }}+V_{\text {normal liver }}},
$$

where $V_{\text {tumor }}$ and $V_{\text {normal liver }}$ indicate the volumes of the tumor and the healthy parenchyma, respectively. For lobar or superselective treatment, the activity is reduced in proportion to the size of the liver volume being treated.

The prescribed activity is reduced by 20 or $40 \%$ if there is an LSF between 10 and $15 \%$ or 15 and $20 \%$, respectively. An LSF higher than $20 \%$ is a contraindication for the treatment [14].

A modified BSA method was used for the SIRFLOX, FOXFIRE, and FOXFIRE-global studies, where activity was reduced relative to the BSA method, based on LSF and tumor involvement [1].

\section{MIRD mono-compartment for glass microspheres}

For glass microspheres, the activity calculation is based on the desired mean absorbed dose to the target liver mass (independent of tumor burden), following:

$$
A[\mathrm{GBq}]=\frac{\text { Desired dose }[\mathrm{Gy}] \times M_{\text {target }}[\mathrm{kg}]}{50[\mathrm{~J} / \mathrm{GBq}]} .
$$

The desired absorbed dose is set assuming a completely homogeneous distribution of the microspheres over the target volume. The target mass may be determined using either CT, MRI, PET, or ${ }^{99 \mathrm{~m}}$ Tc-MAA SPECT [21].

The recommended absorbed dose ranges from 80 to $150 \mathrm{~Gy}$, depending on the judgment of the treating physician. The estimated total activity shunting to the lungs should not exceed $610 \mathrm{MBq}$, which equates to approximately 30 Gy in $1 \mathrm{~kg}$ lung tissue [21]. 


\section{MIRD mono-compartment method for holmium microspheres}

For the administration of holmium microspheres, a methodology akin to the MIRD mono-compartment method for glass microspheres was used in a phase I absorbed dose-escalation study [24]. The administered activity was calculated according to

$$
A[\mathrm{GBq}]=\frac{\text { Liver dose }[\mathrm{Gy}] \times M_{\text {liver }}[\mathrm{kg}]}{15.9[\mathrm{~J} / \mathrm{GBq}]},
$$

where the liver mass was determined on contrast-enhanced CT. The absorbed dose was escalated from 20 to $80 \mathrm{~Gy}$ in four steps. The maximum tolerated absorbed dose was established to be $60 \mathrm{~Gy}$.

\section{Limitations of current methods}

An obvious limitation of these methods is that the actual spatial dose distribution of an individual patient is neglected. In general, these methods seek to prevent overdosing to the parenchyma (and lungs), minimizing the occurrence of radioembolization-induced liver disease [25-27]. As a consequence, the resultant prescribed activities are likely curbed by toxicity limitations of the most vulnerable patients and the occurrence of patients with a highly unfavorable absorbed dose distribution. This is thought to result in under-dosing in some patients [28-30].

For the BSA method, an added limitation is that the estimated liver volume is based on a healthy population. As such, this relation might not hold for patients with liver tumors. Indeed, it has been shown that absorbed liver dose does not correlate with prescribed activity using the BSA method [31]. This results in patients with relatively small livers that are more likely to be overdosed and patients with larger livers are more likely to be under-dosed (see Fig. 1) [29, 31, 32]. An illustration of this is given in [31] where, based on the BSA method, a patient received $1.82 \mathrm{GBq}$ (BSA $1.78 \mathrm{~m}^{2}$, tumor involvement 15\%), resulting in a high liver absorbed dose of $74.7 \mathrm{~Gy}$, due to its relatively low mass of $1.22 \mathrm{~kg}$. In the same study, another patient received a similar activity of $1.85 \mathrm{GBq}\left(\mathrm{BSA} 1.50 \mathrm{~m}^{2}\right.$, tumor involvement $45 \%$ ), but that patient had a larger liver of $2.33 \mathrm{~kg}$, resulting in a much lower average liver absorbed dose of 39.7 Gy. Furthermore, there are currently no guidelines regarding activity prescription after prior resection [33].
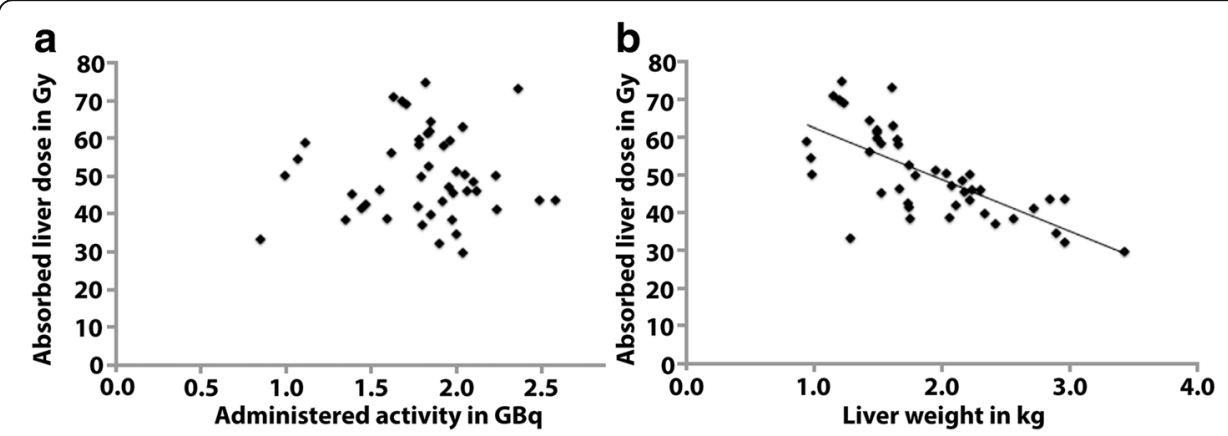

Fig. 1 Adapted from [27]. Absorbed dose to the whole liver was not correlated to the administered activity (a). However, liver weight was negatively correlated with whole liver absorbed dose $(r=-0.723, P<0.001)$, leading to patients with small liver being relatively over-dosed and patients with larger liver under-dosed (b) 


\section{Multi-compartment dosimetry}

A different approach to activity prescription from the homogenous, single compartment models of the BSA and MIRD mono-compartment methods is the partition model (PM). It postulates three compartments with potentially different activity uptakes: tumor, normal liver, and lung tissue [18]. As such, it allows for the selection of a prescribed activity that maximizes the absorbed dose to the tumor tissue, while not exceeding toxicity thresholds for the other two compartments. The expected activities in each compartment are usually based on the distribution of ${ }^{99 m}$ Tc-MAA on the safety scan. However, there is some discussion in the literature about the predictive value of these particles for the subsequent ${ }^{90} \mathrm{Y}$ microsphere distribution [34-37].

The respective compartments are usually segmented on an anatomical imaging modality (e.g., contrast-enhanced CT) or a functional modality (e.g., SPECT thresholding) and registered to the reconstructed ${ }^{99 \mathrm{~m}} \mathrm{Tc}$-MAA distribution. The activity distribution over the compartments is described by the tumor-to-normal tissue ratio (TN ratio), expressed as

$$
\mathrm{TN}=\frac{A_{T}[\mathrm{MBq}] / M_{T}[\mathrm{~kg}]}{A_{\mathrm{NL}}[\mathrm{MBq}] / M_{\mathrm{NL}}[\mathrm{kg}]},
$$

where $A$ and $M$ indicate the activity in and the mass of the tumor $(T)$ and normal liver tissue $(N L)$ compartments.

Using some algebra, the following relation can be derived for the prescribed activity, given a certain TN ratio, LSF and compartment masses [38]:

$$
A[\mathrm{GBq}]=D_{\mathrm{NL}}[\mathrm{Gy}] \frac{\mathrm{TN} \times M_{T}[\mathrm{~kg}]+M_{\mathrm{NL}}[\mathrm{kg}]}{50[\mathrm{~J} / \mathrm{GBq}] \times(1-\mathrm{LSF})},
$$

where $D_{\mathrm{NL}}$ indicates the absorbed dose to the parenchyma. Implicit in this equation is the assumption that dose is deposited locally in the compartment that contains the activity, which is a simplification. This is discussed in further detail in the section "Dosimetric models."

Multi-compartment dosimetry is claimed to be more 'scientifically sound' than the BSA-based or MIRD mono-compartment method [29]. However, besides being more labor intensive to work with in clinical practice, there are several technical caveats to using the PM.

\section{Different methods to calculate TN ratio}

When multiple lesions are present, each may have a different microsphere uptake, leading to errors in the subsequent individual tumor absorbed dose estimates, due to averaging of the TN ratio. Mikell et al. have shown this effect by comparing the silver standard Monte Carlo-based dose estimates with the MIRD mono-compartment based dosimetry and the PM model in realistic patient data [39]. In the case of multiple tumors, there can be large discrepancies between the methods for the estimated tumor absorbed dose. For example, the variability between PM-based and Monte Carlo-based tumor absorbed dose estimates was higher by a factor five in cases where there were multiple tumors present, compared to single tumor samples. 
However, there is currently no consensus on how to calculate the TN ratios for individual tumors for the use in the PM model. Some authors use the entire normal liver volume for this calculation [40], whilst others opt for a smaller sample volume, placed near the tumor-of-interest [36]. Although this simplification makes the use of the PM model more feasible in clinical practice, it also inevitably leads to larger uncertainty $(\approx 2.5 \times)$ in the $\mathrm{TN}$ ratio estimations when the microspheres are not strictly homogenously distributed in the healthy liver tissue [39].

\section{Definition of compartments on anatomical imaging}

When diagnostic (contrast-enhanced) CT or MRI is used for the delineation of the tumor compartments, these delineations subsequently need to be transferred to the SPECT/CT reconstructions. This can be achieved by copying the volume-of-interest (VOI) delineation to the SPECT/CT data or by using (non-rigid) coregistration. However, mismatches are likely to occur, causing a misalignment between the anatomical delineations and the SPECT reconstruction. A common cause is differences in patient positioning between both anatomical scans. For instance, different arm positioning (above the head vs. lying next to the body) or body position (e.g., different placement on the table).

Another issue for coregistration is breathing during the CT acquisition. The acquisition of the liver volume is usually much faster than an entire respiratory period, resulting in a 'snapshot' of a random respiratory phase. As the SPECT or PET activity reconstruction is a superposition of all respiratory phases, this can result in mismatches of $>1 \mathrm{~cm}$ between the anatomical delineations and the reconstructed activity [41]. Using CTs acquired during breath-hold for coregistration might mitigate this effect, but breath-holds are shown to have a limited reproducibility between acquisitions, resulting in different relative respiratory states between scans [42]. A viable solution might be the use of so-called 'time-averaged 3D mid-position CT scans' in this context, often used in radiotherapy [43].

Besides leading to mismatches in coregistration, respiratory motion also results in activity reconstructions that are 'smeared out.' This leads to an underestimation of the local activity concentration, especially in tumor tissue, which has a smaller volume compared to the motion amplitude than the background compartment. The effect of motion blurring is well-known in general [44, 45], but the impact of respiratory motion in the context of radioembolization has recently been shown for both PET [46] and SPECT [47].

Furthermore, defining the boundaries of the tumor compartment on anatomical modalities may be non-trivial in the case of morphologically diffuse or infiltrative tumors [29]. Tumors with substantial necrosis pose a similar problem. A possible solution to this might be the use of FDG PET for the demarcation of vital tumor tissue in the case of FDG-avid tumors.

\section{Definition of compartments using physiological information}

Similarly, the uptake information in SPECT reconstructions (e.g., when using MAA for absorbed dose prediction) could be used to indicate vital tumors. However, as delineations drawn directly on SPECT will generally result in errors in the estimated volume 
[48-50] (Fig. 2), Garin et al. have developed a hybrid method in which the SPECT reconstruction and CT information are presented in conjunction, integrating functional and anatomical information and aiding manual delineation [51,52]. This has been shown to work well for both phantom and patient studies, in which anatomical borders are readily discernable. However, this type of method does not have a well-defined contouring guideline, which reduces reproducibility.

A more fundamental approach to this segmentation problem was proposed in a study by Lam et al. [53], in which directly after the normal ${ }^{99 m}$ Tc-MAA SPECT scan, the participating patients were injected with ${ }^{99 \mathrm{~m}}$ Tc-sulfur colloid (SC) and another SPECT was acquired after $5 \mathrm{~min}$. This compound specifically accumulates in functional (non-tumor) liver tissue and as such will act as a negative template for the tumor compartments. By taking the difference between the MAA and SC SPECT reconstructions, voxel maps for healthy parenchyma and tumor tissue are automatically obtained, providing a 'physiology-based segmentation.'

\section{Voxel-based dosimetry}

In voxel-based dosimetry, the reconstructed voxel is taken as the smallest independent spatial unit for activity. This allows for the expression of (estimated) absorbed dose gradients and non-homogeneities on a small spatial scale, somewhat similar to external beam radiotherapy (EBRT). This contrasts with multi-compartment models, where absorbed dose estimates are averaged over each compartment. By including this spatial dimension, voxel-based dosimetry

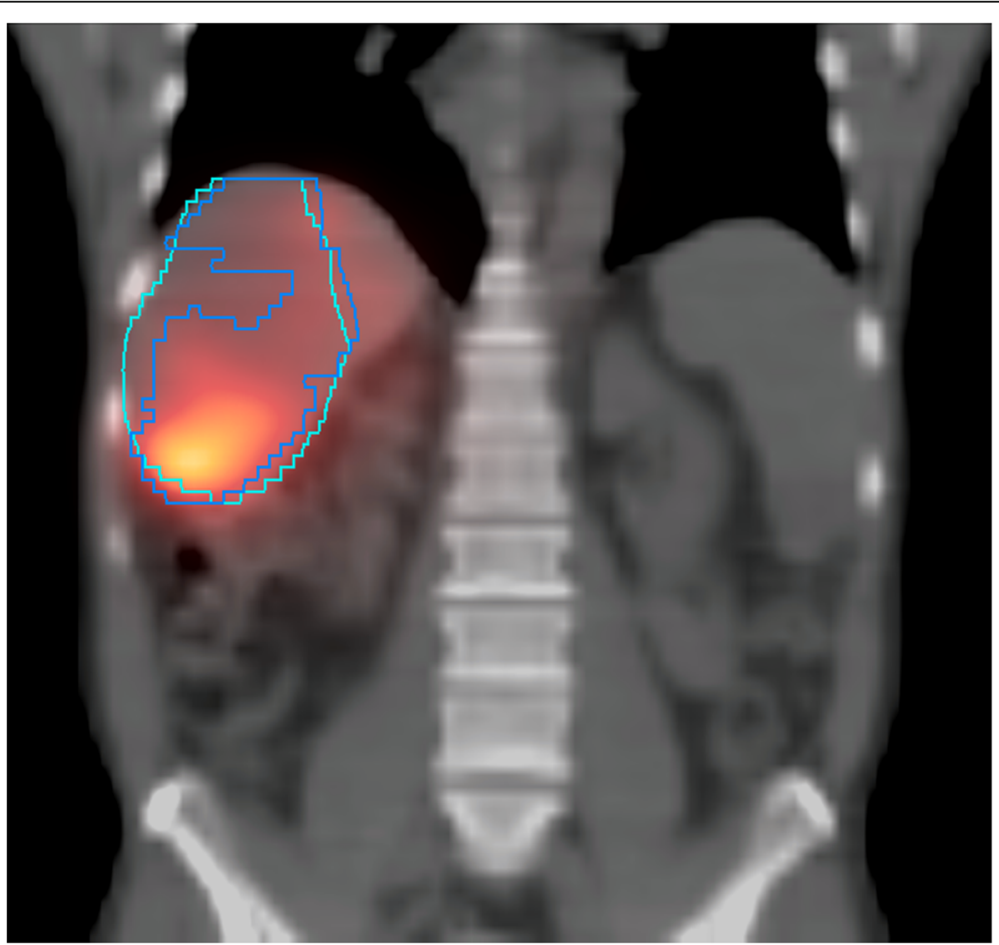

Fig. 2 Exemplar case where a VOI delineation based on SPECT thresholding only (blue contour) does not match the CT-based anatomical tumor definition (teal contour). The mismatch results in a difference in tumor volume and mean tumor uptake 
potentially provides a link to the rich EBRT literature on dose-effect relationships, which could potentially be used for both therapy planning and post-therapy outcome assessment. However, in contrast to image-guided absorbed dose planning for EBRT, voxel-based dosimetry for radioembolization is based on nuclear medicine images, which are generally noisy and of low resolution, prohibiting a direct translation of EBRT concepts to the radioembolization paradigm.

\section{Using spatial dose information}

To aid assessment and comparison between individual cases, the spatial dose information can be combined into a (cumulative) dose-volume histogram (cDVH). These graphs express the fraction of the total VOI (be it a tumor, normal tissue, or entire liver) receiving a certain minimum absorbed dose. This expresses in a single graph how the absorbed dose is distributed over the volume (Fig. 4). The concept of cDVHs also enables the introduction of spatially dependent measures of absorbed dose such as $D_{70}$ (minimum absorbed dose to $70 \%$ of VOI) and $V_{100}$ (percentage of VOI receiving at least $100 \mathrm{~Gy}$ ) that might be expected to be good predictors of treatment effect [54, 55]. For example, it is clear from Fig. 4a that the blue absorbed dose distribution clearly delivers a higher absorbed dose to more of the tumor volume (or conversely, red is less toxic when this is a cDVH of normal tissue). These metrics are widely used for the comparison between EBRT plans and are gaining some traction within the radioembolization dosimetry community to help better explain clinical outcomes [54, 56-59] (see "Dose-effect relationships" section).

Due to the typical heterogeneous distribution of the microspheres, comparing cDVHs of, for instance, two patients is not always trivial (as is the case in Fig. 4a). Ambiguity can occur and an example of such a case is shown in Fig. 4b, where the cDVH curves are crossing. In such cases, it is completely dependent on the specific organ (e.g., parallel organ or not) which cDVH would lead to the highest tumor kill or least amount of toxicity.

This ambiguity is a well-known phenomenon in EBRT, and efforts have been undertaken to create radiobiological models that aim to quantify the biological effect of any treatment plan and enable the comparison of plans based on expected outcome [60]. The premise of most of these models is that an irradiated tumor exhibits a binary response (control or survival), which is determined by the surviving fraction (SF) of a population of cells after irradiation. This SF is modeled as a function of absorbed dose and may include any additional clinically relevant parameters, such as repopulation between treatments, clonogen radioresistance, and dose rate effects. Subsequently, the parameters of these models are retrospectively fitted on clinical data and can then be used to predict treatment outcome. As such, these radiobiological models provide a link between physical quantities such as spatial dose distribution and expected clinical outcome. The potential importance of radiobiological modeling is illustrated with a clinical example Fig. 3.

Importantly, two such models have been adapted from EBRT for the context of radioembolization [56, 61]. First, the effect of dose rate and cell repair mechanisms can be modeled with the biologically effective dose (BED), such that $\ln (\mathrm{SF})=-\alpha$ BED. BED can be calculated for a unit volume $i$ (e.g. a voxel) according to 

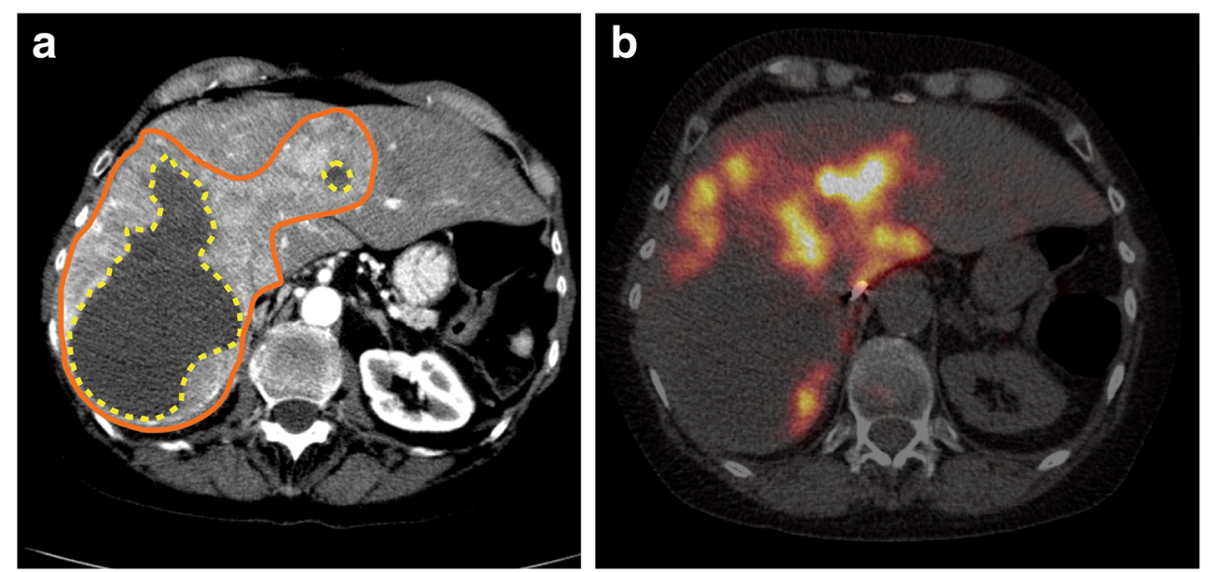

Fig. 3 Example of a large neuroendocrine tumor, which was treated with glass microspheres. Activity was prescribed according to the MIRD mono-compartment method to reach $120 \mathrm{~Gy}$. According to the PM model, the average absorbed dose to the tumor was $150 \mathrm{~Gy}$. The patient has shown no response after treatment (RECIST, mRECIST, and EASL). The contrast-enhanced CT shows the tumor as a large enhanced area (orange solid line) and necrosis (yellow dotted line) (a). A strong absorbed dose inhomogeneity can be observed (b). Voxel-based dosimetry and radiobiological models may account for such absorbed dose inhomogeneities

$$
\mathrm{BED}_{i}=D_{i}\left(1+\frac{D_{i} \cdot T_{\text {rep }}}{\left(T_{\text {rep }}+T_{\text {phys }}\right) \cdot \alpha / \beta}\right),
$$

with $D_{i}$ the locally absorbed dose, $T_{\text {rep }}$ and $T_{\text {phys }}$ the halftimes for cell repair after damage and the physical halftime of ${ }^{90} \mathrm{Y}$, respectively. $\alpha$ and $\beta$ denote the so-called intrinsic radio sensitivity and potential sparing capacity [62].

Furthermore, spatial non-uniformities can be normalized to a single number, called equivalent uniform biologically effective dose (EUBED), see also Fig. 4c. This number is
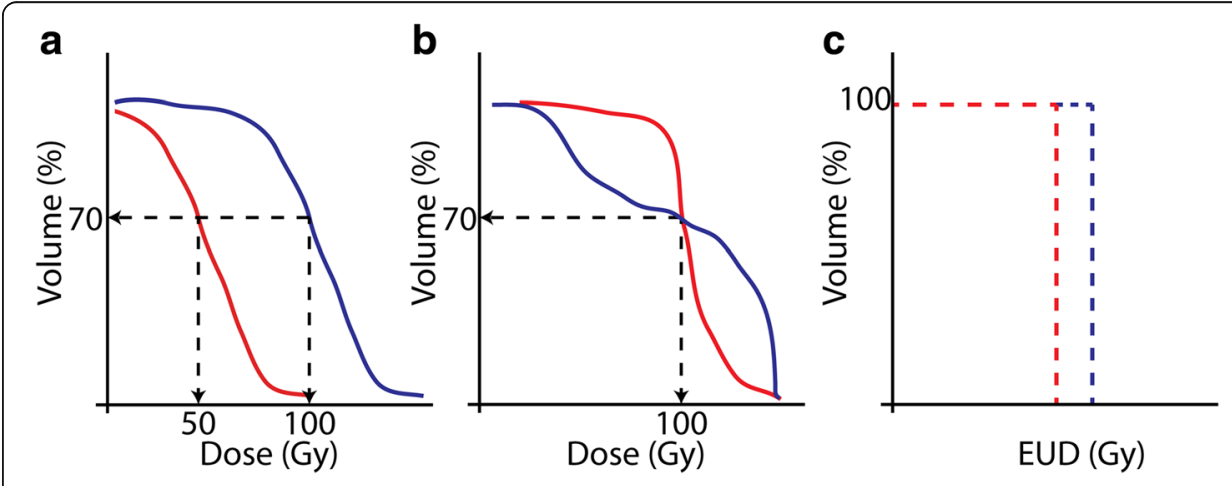

Fig. 4 Hypothetical cDVHs illustrating key concepts in voxel-based dosimetry which may be used for outcome prediction. In panel (a) the situation of the red absorbed dose distribution may be expected to have a smaller impact on the tissue under consideration (less toxic or less tumor kill). This is also reflected in the $D_{70}$ and $V_{100}$ being lower for the red than that for the blue curve. Due to highly heterogeneous absorbed dose distributions, which is typical for radioembolization, two different cases with CDVHs as depicted in panel $\mathbf{b}$ might occur. Which of these cDVHs may be expected to have a larger effect on the tissue, is ambiguous (same $D_{70}$ and $V_{100}$ ) and might depend on the tissue type. c Depicts the hypothetical differences in equivalent uniform doses (EUD), derived from the situation in panel $\mathbf{b}$, potentially resolving the ambiguity 
the same for different absorbed dose distributions that have the same biological effect [63]. EUBED can be defined as

$$
\mathrm{EUBED}=-\frac{1}{\alpha} \ln \left(\frac{\sum_{\mathrm{i}} \mathrm{e}^{-\alpha \mathrm{BED}_{i}}}{n_{\mathrm{voxel}}}\right),
$$

Where $\alpha$ is the radiosensitivity (1/Gy) of the local tissue, $n_{\text {voxel }}$ is the number of voxels of the current VOI, and $i$ denotes the voxel index [61]. A reasonable simplification for radioembolization is to neglect quadratic effects (i.e., $\beta=0$ ) and BED, in which case $\mathrm{BED}_{i}$ is substituted with $D_{i}$ in Eq. 8, which then yields equivalent uniform dose (EUD).

In theory, this approach will aid physicians to optimally weigh risks and benefits of an individual absorbed dose distribution, as clinical outcomes can be linked to a single number such as BED and EUBED. However, the existence and robustness of such dose-effect relationships in the context of radioembolization are currently still under investigation.

\section{Dose-effect relationships}

There is an increasing literature on dose-effect relationships in radioembolization that utilizes advanced dosimetry. An overview of recent papers that (implicitly) estimate tumor control probability (TCP) and/or non-tumor complication probability (NTCP) is given in Table 1. The search for the combination of tumor type, outcome measure, dosimetric model, and imaging modality that yields the best predictive power is very early stage. Consequently, there is a wide variety in each of these properties amongst these studies, resulting in diffuse optimal absorbed dose limits for both liver complications ( 50-97 Gy) and tumor control ( 50-560 Gy). Four major factors are hypothesized to contribute to this: differences in response measures, absorbed dose calculations, microsphere type, scan modality (including acquisition and reconstruction settings), and tumor type.

\section{Response measures}

In these studies, tumor response is assessed according to RECIST, mRECIST, vRECIST, EASL, densitometric change [64], change in total lesion glycolysis (TLG), or standardized uptake value (SUV). What is considered a complete response, partial response, stable disease, or progressive disease differs significantly between these measures [65]. For example, the RECIST criteria are sensitive to changes in tumor size, whereas TLG expresses (changes in) total glycolysis (tumor volume times mean SUV over the VOI). Consequently, minimum absorbed dose estimates that lead to tumor response are different between criteria. Although some attempts have been made to directly compare some of these methods [66], the use of such a variety of methods makes comparing these data non-trivial, if not impossible. As the most relevant clinical outcomes are overall survival and progression-free survival, it is important to establish which of the reported proxies is the most predictive of survival [67]. This may result in disease-specific outcome measures (e.g., EASL or mRECIST for hepatocellular carcinoma and RECIST or TLG for metastatic colorectal cancer).

Some studies that are reported in Table 1 incorporate either metabolism-based (functional) masks from a previous FDG PET [55] or, for example, the $D_{70}$ measure [54]. However, most studies calculate the average absorbed dose to the tumor. This may 
disregard the existence of necrotic volumes and, more generally, absorbed dose heterogeneity.

\section{Absorbed dose calculations}

Which absorbed dose calculation method best reflects the underlying radiobiological processes in the entire patient population remains an open question. Theoretically, applying EUD and/or BED-based models should be best suited to naturally incorporate differences in specific activity and absorbed dose heterogeneity in a tissue, as described above. But clinically, a clear advantage over average absorbed dose to the tumor is yet to be found $[56,61,68,69]$. The authors suggest this might be linked to the outcome measure being too crudely categorized [61]. Another central finding in these studies, however, is that the apparent radio sensitivities ( $\alpha$ and $\beta$ in Eq. 7) of both the tumor and hepatic tissues in radioembolization are an order-of-magnitude lower than what is found in the EBRT setting, even when correcting for absorbed dose inhomogeneities [61]. Moreover, a significant difference between the absorbed dose needed to reach $\mathrm{TCP}(50 \%)$ for glass and for resin microspheres has been found [61, 68]. In conclusion, the values of the relevant parameters in the radiobiological models have not been well established for radioembolization. They may be specific to the type of microsphere and it can even be expected to be different between ${ }^{90} \mathrm{Y}$ and ${ }^{166} \mathrm{Ho}$-based microspheres. These uncertainties have a direct impact on the determination of BED and EUD.

\section{Micro-distribution}

A possible explanation for the differences found between glass and resin microsphere dose-effect relationships is the potential difference in micro-distribution.

One of the first papers on in vivo microsphere distribution was by Fox et al. [70]. Using a beta-probe, they showed that the activity pattern on a sub-centimeter scale was highly heterogeneous. Later, Yorke et al. [71] used a combination of computer simulations and biopsy samples to try to find an explanation for the clinically observed lack in normal liver complications using glass microspheres at absorbed dose levels that are known to cause complications in EBRT and found that absorbed dose heterogeneity is sufficient to explain this incongruity.

More recently, Walrand et al. performed a simulation study of normal liver tissue, finding that the relatively low number of injected glass microspheres results in non-uniform trapping in the terminal portal artery, resulting in tissue volumes receiving sub-lethal absorbed doses. This would both explain the relatively low toxicity per Gray of glass, relative to resin microsphere and the granularity observed in post-treatment ${ }^{90}$ Y PET (see Fig. 5) [72].

This conclusion was seemingly contradicted in an elaborate histological study by Högberg et al., who found that a higher concentration of microspheres (i.e., in the case of resin microspheres) leads to a higher tendency to form clusters, especially in the larger (upstream) arterioles, resulting in a more non-uniform absorbed dose distribution in the liver parenchyma [73]. According to these authors, this apparent contradiction stems from the fact that Walrand et al. only assumed microsphere trapping in the terminal branches of the infused artery. In a subsequent simulation study, Högberg et al. were able to replicate their histological findings in a mathematical model. This places 


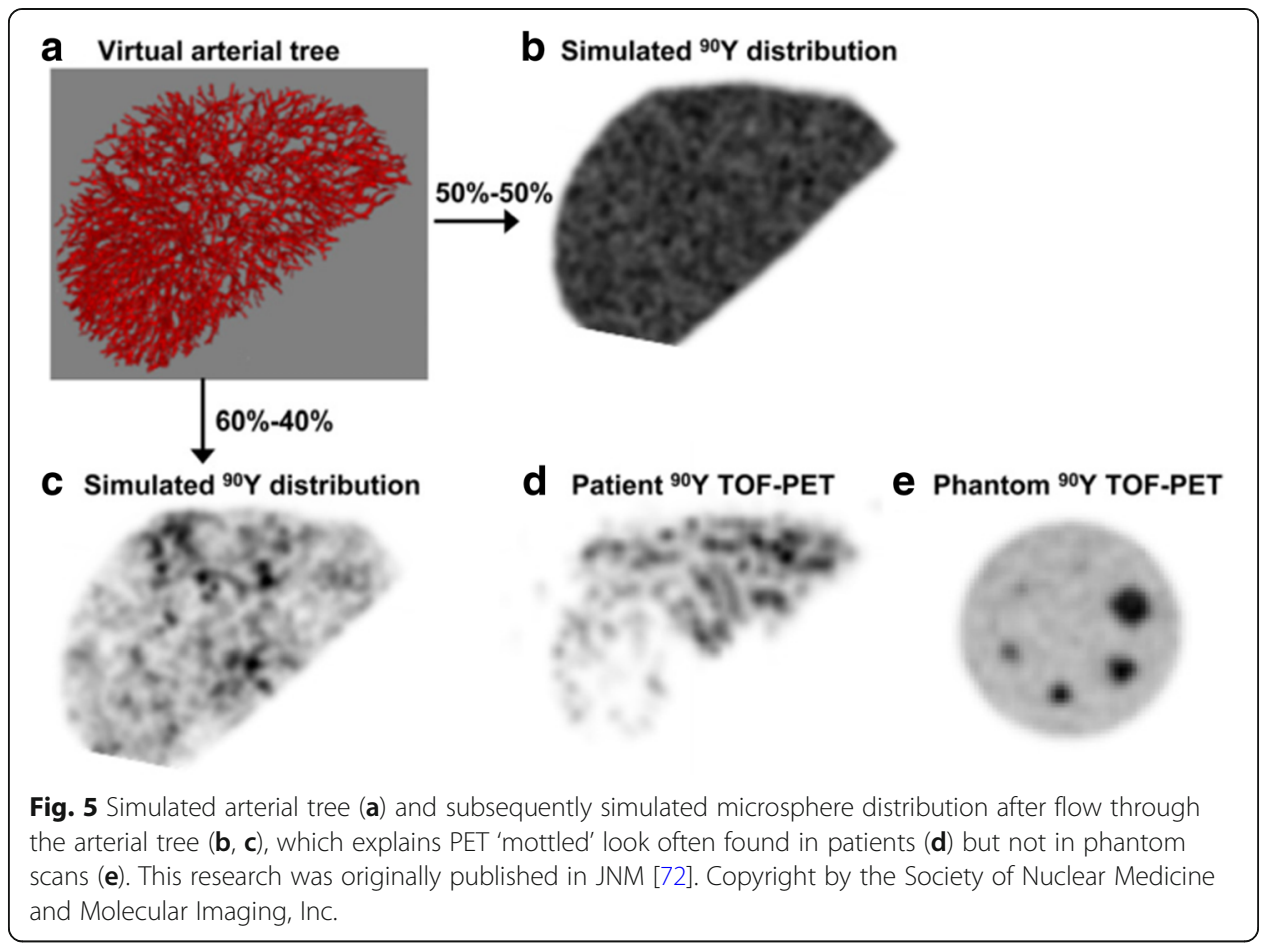

further emphasis on the importance of the geometry of the arterial tree and (local) microsphere concentration as drivers for microsphere distribution inhomogeneity [74]. These models, however, predict cluster propensity as a function of arteriole generation (branch number) and lack further spatial information. Consequently, the authors conclude that the micro-scale clusterings they observed in itself might not (fully) explain the observed macroscopic inhomogeneities, as measured by non-invasive imaging [73].

Pasciak et al. tried to bridge the gap between micro- and macro-scale tumor dosimetry by using Monte Carlo-based estimations of microsphere micro-distributions, given a ${ }^{90} \mathrm{Y}$ PET reconstruction of a patient [75]. These microsphere micro-distributions are simulated by drawing properties such as cluster propensity and distance from probability density functions that were constructed from histological data [76]. This resulted in realistic structures (Fig. 6) such as clusters and strings of microspheres. Crucially, it provides a plausible link between the observations in macro- and microdosimetry.

\section{Quantitative image reconstruction}

Besides the abovementioned factors concerning dosimetry, differences in outcome between these studies may in part also be explained by the wide range in technical scan parameters used and the measurement variance inherent to nuclear medicine images. An overview of the current topics in nuclear image acquisition and reconstruction is therefore desired.

In quantitative image reconstruction, all relevant interactions between the radionuclide, the patient, and the imaging system need to be accounted for during reconstruction. The current state-of-the-art consists of iterative reconstruction algorithms that incorporate models for all such image-degrading effects (e.g., attenuation, scatter, nuclide decay, detector uniformity) [77-79]. 

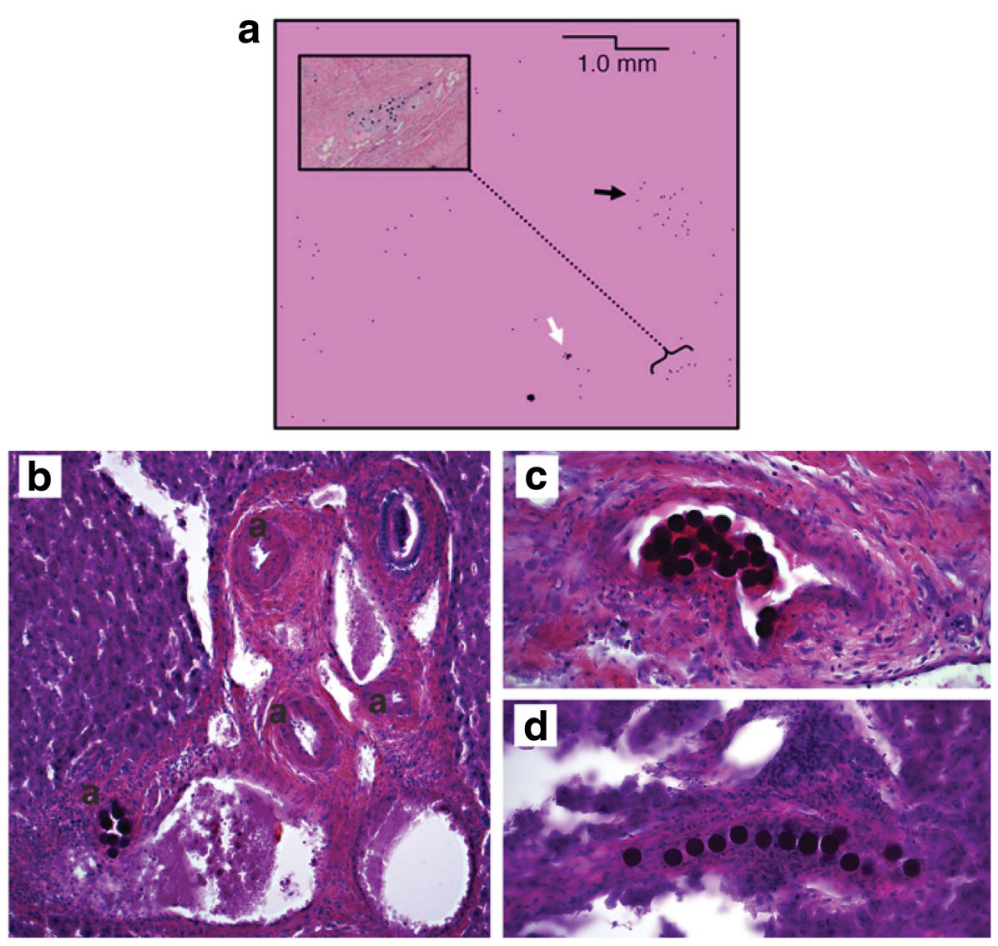

Fig. 6 a Small clusters (white arrow) and large clusters (black arrow) are apparent in the Monte Carlo simulations by Pasciak. These simulated distributions seem to be consistent with the histological findings of (amongst others) Högberg (b, c, d). Panel a was originally published in JNM [75]. Copyright by the Society of Nuclear Medicine and Molecular Imaging, Inc. Other panels are adapted from [74]

From its inception, PET was considered a quantitative modality, in contrast to SPECT. This is due to the high signal-to-noise ratio of PET and the relative simplicity of the physics of coincidences, which enables a straight-forward method for attenuation correction. This was available in early generations of PET scanners. However, with the advent of inherently coregistered CT in SPECT/CT, attenuation and scatter correction are now common practice and some authors have claimed that modern clinical SPECT systems can now be considered quantitative as well [80, 81]. Furthermore, vendors are currently implementing calibration routines and inherently quantitative reconstruction software in their machines [82, 83], which enables the dissemination of absolute activity quantification into clinical practice for both modalities.

\section{Post-therapy imaging}

In radioembolization, treatment success can be assessed with a post-therapy scan, either with bremsstrahlung SPECT/CT (bSPECT) or PET/CT $\left({ }^{90} \mathrm{Y}\right.$ PET).

Bremsstrahlung SPECT/CT - the relevance of physics modeling Post-therapy assessment with ${ }^{90} \mathrm{Y}$ may be performed by bremsstrahlung imaging. This is different from mono-energetic emitters (such as ${ }^{99 \mathrm{~m}} \mathrm{Tc}$ ) in that ${ }^{90} \mathrm{Y}$ produces a broad and continuous energy spectrum without a photopeak. Secondly, the high flux of bremsstrahlung photons on a gamma camera will result in significant dead time, if not managed correctly (count rate linearity was estimated up-to $7.5 \mathrm{GBq}$ for ${ }^{90} \mathrm{Y}$ and $1.5 \mathrm{GBq}$ 
for ${ }^{166} \mathrm{Ho}$ [84]). Therefore, ${ }^{90} \mathrm{Y}$ image quantitation using a gamma camera was recognized early on as being non-trivial [85]. With the advent of advanced iterative reconstruction techniques that enable advanced physics modeling, several quantitative reconstruction methods have been proposed in the literature. Most of these methods utilize some kind of Monte Carlo modeling of the imaging process. Rong et al. achieved quantitation errors between -1.6 and $11.9 \%$ for a phantom experiment by modeling all relevant energy-dependent image degrading effects [86]. Elschot et al. incorporated Monte Carlo simulations of photon-tissue interactions directly within the iterative reconstruction loop, increasing image contrast and activity recovery significantly (over $80 \%$ for non-small spheres), relative to a reference clinical reconstruction algorithm [87]. Minarik et al. performed a similar study, using the SIMIND code and achieved a quantification error around $+8.5 \%[88]$.

However, these methods rely on advanced Monte Carlo techniques, which are currently not easily accessible for institutions without a medical physics team that has extensive experience with these methods. Consequently, the reported accuracies will be significantly worse in normal clinical practice.

${ }^{90} \mathrm{Y}$ PET - the impact of machine and reconstruction parameters ${ }^{90} \mathrm{Y}$ can also be imaged using PET. However, as ${ }^{90} \mathrm{Y}$ only has a minute positron branching ratio ( $32 \mathrm{ppm}$ ) and the detectors were expected to be saturated from the bremsstrahlung photons (which was later demonstrated to be false), for a long time, PET was not considered a feasible modality for post-therapy imaging. The earliest in vivo demonstration of the feasibility of ${ }^{90} \mathrm{Y}$ PET imaging was delivered by Lhommel et al. in 2009 [89] using a time-of-flight (TOF) PET scanner and an additional copper ring inserted in the gantry to prevent detector saturation. Later, the feasibility of ${ }^{90} \mathrm{Y}$ PET with Lutetium oxyorthosilicate (LSO) crystals in a scanner without TOF capability was demonstrated [90].

These initial proofs-of-concept were followed by studies that corroborated the quantitative reconstruction capabilities of ${ }^{90} \mathrm{Y}$ PET, using clinically available methods [91-94] which were applied to clinical data [54, 95-97]. However, the very high contribution of randoms (>90\%) due to bremsstrahlung in combination with the very low coincidence count statistics was expected to impact random coincidence estimation, scatter correction, and consequently, image quality. An elaborate study by Carlier et al. has shown that the effect of these phenomena on bias, variability, and detectability of hotspots is minor. The use of correct point spread function (PSF) modeling and TOF reconstruction kept background variability and noise at acceptable levels [98]. This was further corroborated in a fully Monte Carlo-based simulation in which ${ }^{90} \mathrm{Y}$ quantitation is compared to that of ${ }^{18} \mathrm{~F}$ [99]. It was found that, relative to ${ }^{18} \mathrm{~F}$, the image quality was only slightly poorer in ${ }^{90} \mathrm{Y}$ for a similar positron emission rate. Furthermore, image quality was not strongly linked to any particular physical effect or reconstruction step. This led to the conclusion that adding ${ }^{90} \mathrm{Y}$-specific models to the PET imaging process is not needed. Furthermore, Van Elmbt et al. have shown that systems based on modern crystals (post-BGO) can be used for ${ }^{90} \mathrm{Y}$ dosimetry [100]. Since then, ${ }^{90} \mathrm{Y}$ image quantification has also been shown to be possible in PET/MRI [101] and solid-state digital PET/CT [94]. 
For clinical ${ }^{18}$ FDG PET, the importance of homogenization of acquisition and reconstruction settings over centers to allow pooling and comparison of data sets is well-recognized and has resulted in the EARL guidelines and accreditation program [102]. A similar initial attempt for ${ }^{90} \mathrm{Y}$ PET has been made in the form of the QUEST study, showing that ${ }^{90} \mathrm{Y}$ PET-based dosimetry should be reproducible across scanners and centers, as long as TOF-capabilities are available [103].

Together, these studies show that PET-based ${ }^{90} \mathrm{Y}$ quantitative imaging is feasible, robust, and straight-forward to implement in clinical practice when a reasonably modern PET system with TOF-capabilities is available. This is in contrast to bSPECT/CT, for which no sufficiently accurate reconstruction methods are currently available for general clinical use.

${ }^{90} \mathrm{Y}$ PET vs. bSPECT In a direct comparison between bSPECT/CT and ${ }^{90} \mathrm{Y}$ PET/CT, the latter is found to have a higher resolution and less scatter in patient studies and several case series $[104,105]$. In a quantitative direct comparison between a state-of-the-art clinical bSPECT/CT reconstruction algorithm and clinical PET/CT reconstruction protocols, the superior contrast, detectability, and absorbed dose estimates of PET were demonstrated [106]. However, this comes at the price of a relatively long scan duration in the case of ${ }^{90} \mathrm{Y}$ PET, which is 15 to 20 min per bed position. When advanced photon-tissue and photon-detector interactions were modeled with a Monte Carlo-based SPECT/CT reconstructor, image contrast improved substantially and was in some cases (in larger hot spots) higher than in PET/CT [87].

In general, it should be noted that currently there is no standardized approach for post-therapy imaging in terms of acquisition and reconstruction settings and there may exist some systematic biases between the various approaches of different groups, even within the same modality. As a consequence, interpreting and comparing dosimetric results between different groups should be done with caution. However, in general, ${ }^{90} \mathrm{Y}$ PET is currently superior to clinical bSPECT/CT in terms of resolution, accuracy, and the clinical availability of accurate reconstruction methods for dosimetry.

MR and CT for ${ }^{166} \mathrm{Ho}$ In contrast to ${ }^{90}$ Y-based microspheres, ${ }^{166} \mathrm{Ho}$ does emit photons with discrete energies that are directly detectable with a gamma camera. Furthermore, it is a paramagnetic element, enabling the visualization with MRI, and it has a very high X-ray attenuation, resulting in good contrast on CT [107, 108]. A quantitative SPECT/CT reconstruction using advanced Monte Carlo-based techniques has been developed [109] (achieving contrast recovery of over 80\% in non-small NEMA spheres), as is a hybrid method to correct for photon down-scatter from bremsstrahlung and higher energy photons [110]. In a direct comparison between SPECT- and MR-based quantification [108], both modalities are found to be suited for peri-therapy dosimetry [111].

\section{Dosimetric models}

With quantitative imaging, the physical quantity activity (i.e., Becquerel or Curie) of the isotope distributed in space is estimated. However, especially in the case of radionuclide therapies, the process of interest is not the activity per se, but rather the subsequent dose absorption by the surrounding tissue (in Gray), as a result of high energy particles 
(betas and photons) emitted in the process of decay. This process of dose absorption is what causes the tumor kill and constitutes a rather complex interaction, which depends both on the tissue and the specific emissions from the isotope.

If the isotope distribution is known exactly, the most comprehensive and precise estimations of absorbed dose are achieved through Monte Carlo simulations of all relevant interactions between the high energy particles and the healthy or tumor tissue. Popular codes include the EGSnrc code [112], MCNP [113], FLUKA [114], and the GATE extension of GEANT [115].

However, these types of simulations are rather complex and time-consuming. Furthermore, the liver is a rather homogenous medium in terms of dose absorption at energies typical for radioembolization. Therefore, a frequently used method to speed up these calculations is by pre-calculating a dose point-kernel (DPK) or dose voxel-kernel (DVK), which is energy absorption in a homogeneous medium around a point source or a voxel source, respectively. Then, a convolution of the true activity distribution with the DPK/DVK will result in an accurate absorbed dose estimation for a homogeneous medium. This kernel can also be scaled to different local tissue densities [116].

The largest contribution to the total absorbed dose comes from the emitted beta particles. The maximal range for ${ }^{90} \mathrm{Y}$ betas in tissue is $1.2 \mathrm{~cm}\left(0.9 \mathrm{~cm}\right.$ for $\left.{ }^{166} \mathrm{Ho}\right)$, which is in the same order of magnitude as the resolution of both SPECT and PET. This implies that most of the energy is deposited within the voxel of origin. Consequently, a further simplification is to assume that all emitted energy is absorbed locally, which is usually called the local deposition model (LDM). In practice, this method constitutes applying an appropriate scaling factor to the voxel values of a quantitative reconstruction.

In a direct comparison of SPECT-based ${ }^{90} \mathrm{Y}$ dosimetry Monte Carlo, DVK and LDM-based dosimetry are found to be nearly identical for activity which is not close to tissue inhomogeneities (e.g., liver-lung border) for the liver [117]. For lung tissue, it was necessary for the DVK method to be scaled to the lower local tissue density of the lung tissue to reach adequate results.

This lack of difference between LDM and the other models is likely to be explained by the fact that a SPECT-based reconstruction has a resolution that is in the same order as the average beta-range. This can be understood as a convolution of a 'blurring' kernel with a putative perfect activity distribution, obviating the need for the simulation of the beta-transport (i.e., blurring). LDM does not unnecessarily repeat this step. Indeed, Pasciak and Erwin found for ${ }^{99 \mathrm{~m}}$ Tc-MAA SPECT reconstructions that LDM outperformed a Monte Carlo-based absorbed dose estimation, due to this effect [118]. Later, this finding was repeated in ${ }^{90} \mathrm{Y}$ PET [119]. Although in most cases for PET, much of the theoretical benefit is obscured due to image noise, causing both techniques to have a similar absorbed dose uncertainty. Still, the authors recommend using the LDM in post-radioembolization ${ }^{90} \mathrm{Y}$ PET dosimetry due to its accuracy and ease-of-use [119].

\section{Timing of dosimetry-based treatment planning}

Pre- and post-treatment are not the only time points for dosimetry, as Bourgeois et al. report on intra-procedural PET/CT in a case study [120]. They used a 3-step protocol wherein the first step ${ }^{90} \mathrm{Y}$ microspheres with a total activity determined by the BSA 
model were administered to the patient. For the second step, the patient was transferred for PET/CT imaging. The maximum absorbed dose by normal hepatic parenchyma and the average absorbed dose by the tumor were determined. For one out of the six patients in this study, the absorbed dose by the tumor was below the assumed tumoricidal absorbed dose of 100-120 Gy for HCC (the other five patients reached this threshold with the first infusion, which was based on the BSA model). For this single, undertreated patient, the third step of the protocol was performed which was a repeated infusion of ${ }^{90} \mathrm{Y}$ microspheres with an optimized activity determined from the quantitative PET/CT data to reach the target tumor absorbed dose. Although the initial treatment planning was based on the suboptimal BSA method, the dosimetry based on the intra-procedural PET/CT scan allowed for activity delivery based on patient-specific physiology at the time of the procedure. The downsides of this 3-step protocol are the increased time, costs, and access to equipment and personnel.

This last disadvantage might be partly solved by imaging in the intervention room. Walrand et al. describe a camera dedicated to bremsstrahlung SPECT of ${ }^{90} \mathrm{Y}$ [121]. They suggested mounting the gamma camera on a robotic arm to allow SPECT acquisition within a few minutes in the intervention room during the catheterization procedure to optimize the ${ }^{90} \mathrm{Y}$ activity to inject.

Another option for imaging in the intervention room is proposed by Beijst et al. [122]. The authors propose a hybrid imaging system, consisting of an X-ray c-arm combined with gamma imaging capabilities for simultaneous real-time fluoroscopic and nuclear imaging. A slightly modified version of this prototype [123] was shown to be able to accurately estimate LSF of a ${ }^{99 m}$ Tc-MAA scout dose in an interventional setting [124]. When this hybrid imaging modality becomes available in the angiography room, it may be possible to move towards 1-day procedures by combining scout and therapy dose in one session.

Using microspheres labeled with a paramagnetic element, like ${ }^{166} \mathrm{Ho}$, will provide contrast on MRI. It has been shown that the absorbed dose by the tumor and healthy liver can be accurately quantified using a post-treatment MRI scan [108, 112]. Since MRI provides excellent soft tissue contrast, it would be a well-suited modality for radioembolization guidance as well as evaluation of therapy. The feasibility of fully MR-guided real-time navigation of hepatic catheterization was demonstrated in an animal model [125]. Drawbacks of MR-guided radioembolization are the potentially limited availability of MR scanners and MR-compatible catheters, and guide wires and the relatively high costs.

\section{Discussion}

Recently, several phase III trials failed to show an improvement in progression-free survival and overall survival when radioembolization with SIR-Spheres was combined with first-line treatments. A reason for this might be that the methods for activity prescription which were used in these studies (BSA and MIRD mono-compartment) are barely personalized and are geared towards safety rather than efficacy. More personalized methods (e.g., the partition model, cDVH-based methods) are available. However, there is no consensus as to what absorbed dose thresholds should be prescribed. In this manuscript, we have therefore reviewed the specific shortcomings of the current 
activity prescription methods and the current state-of-the-art of newer dosimetric methods and understanding of the underlying radiobiology.

Currently, there is a large range in the literature regarding dosimetric limits, both for the TCP and the NTCP (Table 1). We believe that one of the biggest drivers of these diffuse limits is the corresponding wide range in modalities, technical settings, analysis, clinical outcome measures, and relatively small sample sizes. It is therefore nearly impossible to compare data from different studies and distill a common absorbed dose limit, regardless of dosimetric method. This also highlights the importance of investigators providing clear and detailed information on the dosimetric method and analysis used in their publication. This will facilitate reproducibility and may allow for the pooling of clinical data.

With the advent of advanced iterative reconstruction techniques, image quality has improved dramatically in both PET and SPECT [80]. This is mainly due to the incorporation of models for the physics of image formation. In contrast to PET and probably owing to the more complex (underdetermined) nature of the physics in SPECT imaging, dissemination of quantitative reconstruction algorithms started only recently for SPECT [84, 126]. For more complex isotopes (e.g., ${ }^{90} \mathrm{Y}$ bremsstrahlung SPECT), this is still in the research phase and vendor-supported solutions are currently not available. The same holds for more complex image-degrading effects (for both SPECT and PET) such as respiratory motion and compensation for partial volume effects. These developments are beneficial to the goal of personalized dosimetry but are currently not widely available.

However, we believe that using the currently available reconstruction techniques, reliable estimates of dosimetric limits may be established. But in order to achieve comparable results, acquisition and reconstruction settings should be standardized. An initiative similar to the EARL accreditation program for ${ }^{18}$ FDG PET should lead to reconstructions that are perhaps less than optimal but are at least comparable between patients and institutions, allowing for the pooling of derived dosimetric data. To illustrate this point, it was recently shown that it is feasible to get reliable absorbed dose estimates from ${ }^{99 \mathrm{~m}} \mathrm{Tc}-\mathrm{MAA}$, even if attenuation correction is lacking, using only a simple calibration [62]. This lowers the technical demands for more personalized dosimetry. For technical parameters in ${ }^{90} \mathrm{Y}$ PET, the QUEST initiative may be regarded as a step in the right direction [103].

Ideally, this standardization should not be limited to technical parameters of a specific imaging modality, but should also include methods for the segmentation of compartments (or voxels of any VOI), the transferring of volumes (e.g., pre-therapy image delineations transferred to post-therapy images), the selection of relevant clinical outcome measures, and stratification by relevant clinical factors (e.g. tumor size, tumor type, baseline liver function). We believe that this standardized acquisition and analysis pipeline may solve the biggest sources of error in current comparative studies (especially multicenter ones). Furthermore, this standardized protocol can be used for prospective studies in dosimetric limits that are relevant to clinicians.

We expect that the formulation of and adherence to such a standardized protocol would be greatly aided if it is based on guidelines formulated by a panel of experts, ideally sponsored by an authoritative entity such as the EANM, SNMMI, or similar.

In order to further refine personalized dosimetry in clinical practice, new methods need to be fast in terms of scan time, labor extensive, robust, and standardized. Some 
examples of developments in this direction are fully data-driven respiratory motion compensation [127, 128] and fast lung absorbed dose estimation [129]. These methods have in common that they are faster, often more reliable and robust and more usable in clinical practice than existing methods. As there is a wide range of clinical parameters that have an influence on response to therapy, we believe that currently the biggest challenge for the medical physics community involved in radioembolization is not the improvement of quantitative imaging in itself, but rather the translation and dissemination of the current state-of-the-art into a usable form for the use in practical dosimetric efforts. The potential increase in clinical workload and costs associated with further refined personalized dosimetry should be weighed against the potential gains and should not a priori be considered a barrier for implementation.

Currently, many aspects of fundamental radiobiology in radioembolization are unknown. For example, the group of Chiesa et al. was unexpectedly unable to show a clear increase in predictive power for outcome when using equivalent uniform dose-based measures, as opposed to the average absorbed dose to the tumor or healthy liver tissue [61]. This illustrates that radiobiological model parameters are not well established for this modality and that the precise relation between absorbed dose non-homogeneity at both the macro (voxel) level and sub-millimeter level and tissue response is not well understood. We expect that a better understanding of radiobiology on this level will aid the establishment of a coherent account on the efficacy of radioembolization and to enable further refinements in patient selection and/or personalized dose optimization. In that sense, the combination of statistical histological data with models that bridge between micro-distribution and clinically observable macroscopic features in reconstructed data (e.g., 'mottled look' in 90Y PET) might provide additional insight into (deviations from) dose-response relationships in a wide population of patients and may result in a micro-scale equivalent uniform dose-metric.

An improved understanding of radiobiology may also facilitate other concepts from EBRT to be translated to the context of radioembolization. An example is fractionation, which uses tumor repopulation and oxygenation between fractions to increase the tumoricidal effect of subsequent irradiations. For radioembolization, this would mean improved tumor control for multiple vs. the current single treatment (e.g., two times 60 Gy vs. 120 Gy at once). Whether or not this effect can be exploited using radioembolization is an area of future research.

A better understanding of the dose-response relationships will lead to an improved selection of patients for which dose may be increased safely. Currently, the only available particle for treatment planning is ${ }^{99 \mathrm{~m}} \mathrm{Tc}-\mathrm{MAA}$, which might be a suboptimal predictor of the subsequent microsphere biodistribution, both in terms of LSF $[15,16]$ and intrahepatic distribution [35-37]. Consequently, a particle that better matches the rheology of the therapeutic microspheres is needed, if radioembolization is to become a true theranostic modality [130]. Several efforts in this direction have been undertaken [131-133]. In this context, ${ }^{166} \mathrm{Ho}$ is a promising alternative in that exactly the same particle can be used for both planning and therapy. This was illustrated for the estimation of LSF [15].

Another approach is to apply dosimetry in an interventional setting [120]. For instance, following an AHASA (as high as safely attainable [8]) paradigm during infusion of the microspheres until thresholds for hepatic toxicity are reached. 
Furthermore, if dosimetry is found to be sufficiently reliable, EBRT could be used after radioembolization on specific target areas that might have received a sub-optimal absorbed dose from radioembolization.

Together, these developments in homogenization, accessibility, and improved methods will ideally lead to the most personalized and optimal treatment, which we expect to result in improved overall survival and progression-free survival.

\section{Conclusions}

A better understanding of dose-response relationships is needed for improved patient selection and dose optimization in radioembolization. To this end, standardization of acquisition, reconstruction, and analysis protocols are needed. Such an effort would greatly benefit from centrally formulated guidelines. This might enable comparison and pooling of clinical data. Disseminating advanced methods from research groups to clinical practice could prove to be useful in this respect.

\footnotetext{
Abbreviations

99mTc-MAA: 99mTc macroaggregated albumin; BED: Biologically effective dose; BSA: Body surface area; bSPECT: Bremsstrahlung SPECT; CDVH: Cumulative dose-volume histogram; Cholangio: Cholangiocarcinoma; CR: Complete response; CRC: Colorectal cancer; DPK: Dose-point kernel; DVK: Dose-voxel kernel; EBRT: External beam radiotherapy; EUD: Equivalent uniform dose; HCC: Hepatocellular carcinoma; LDM: Local deposition model; LMER: Linear mixed-effects regression model; LSF: Lung shunt fraction; NET: Neuroendocrine tumor; NTCP: Non-tumor complication probability; PM: Partition model; PR: Partial response; PSF: Point spread function; ROI: Region-of-interest; SC: Sulfur colloid; SD: Stable disease; SF: Surviving fraction; SUV: Standardized uptake value; TCP: Tumor control probability; TLG: Total lesion glycolysis; TN ratio: Tumor-to-normal tissue ratio; TOF: Time of flight; VOI: Volume-ofinterest

\section{Funding}

This work was supported in part by a research grant from Siemens Medical Solutions (HWAMdJ). Siemens did not participate in the design of the study, the collection, analysis, and interpretation of the data nor in the writing of the manuscript.

This project has received funding from the European Research Council (ERC) under the European Union's Horizon 2020 research and innovation programme (grant agreement no. [646734]).
}

\section{Authors' contributions}

HWAMdJ, MGEHL, and SCK designed the initial outline of the manuscript. RB drafted the manuscript with input from all authors. BK has written a section of the manuscript. AJATB has provided written text as well as clinical examples. All authors read and approved the final manuscript.

Ethics approval and consent to participate

For this type of study, formal consent is not required and informed consent is not applicable.

\section{Consent for publication}

Not applicable

\section{Competing interests}

MGEHL is a consultant for BTG International and Terumo and has received research support from Quirem Medical.The Department of Radiology and Nuclear Medicine of the UMC Utrecht receives royalties from Quirem Medical.

\section{Publisher's Note}

Springer Nature remains neutral with regard to jurisdictional claims in published maps and institutional affiliations.

\section{Author details}

'Department of Radiology and Nuclear Medicine, University Medical Center Utrecht, Room E01.132, P.0. Box 85500, 3508, GA, Utrecht, The Netherlands. '2Department of Imaging Physics, The University of Texas MD Anderson Cancer Center, 1155 Pressler St, Unit 1352, Houston, TX 77030, USA.

Received: 27 November 2017 Accepted: 19 June 2018

Published online: 02 November 2018

\section{References}

1. Gibbs P, Gebski V, Van Buskirk M, Thurston K, Cade DN, Van Hazel GA. Selective internal radiation therapy (SIRT) with yttrium-90 resin microspheres plus standard systemic chemotherapy regimen of FOLFOX versus FOLFOX 
alone as first-line treatment of non-resectable liver metastases from colorectal cancer: the SIRFLOX study. BMC Cancer. 2014;14:897.

2. Dutton SJ, Kenealy N, Love SB, Wasan HS, Sharma RA, FOXFIRE Protocol Development Group and the NCRI Colorectal Clinical Study Group. FOXFIRE protocol: an open-label, randomised, phase III trial of 5-fluorouracil, oxaliplatin and folinic acid (OxMdG) with or without interventional selective internal radiation therapy (SIRT) as first-line treatment for patients with unresectable liver-on. BMC Cancer. 2014;14:497.

3. Van Hazel GA, Heinemann V, Sharma NK, Findlay MPN, Ricke J, Peeters M, et al. SIRFLOX: randomized phase III trial comparing first-line mFOLFOX6 (plus or minus bevacizumab) versus mFOLFOX6 (plus or minus bevacizumab) plus selective internal radiation therapy in patients with metastatic colorectal cancer. J Clin Oncol. 2016;34:1723-31.

4. Wasan HS, Gibbs P, Sharma NK, Taieb J, Heinemann V, Ricke J, et al. First-line selective internal radiotherapy plus chemotherapy versus chemotherapy alone in patients with liver metastases from colorectal cancer (FOXFIRE, SIRFLOX, and FOXFIRE-global): a combined analysis of three multicentre, randomised, phase 3 trials. Lancet Oncol. 2017;18:1159-71.

5. Vilgrain V, Pereira H, Assenat E, Guiu B, llonca AD, Pageaux GP, et al. Efficacy and safety of selective internal radiotherapy with yttrium-90 resin microspheres compared with sorafenib in locally advanced and inoperable hepatocellular carcinoma (SARAH): an open-label randomised controlled phase 3 trial. Lancet Oncol. 2017;18:1624-36.

6. Chow PKH, Gandhi M, Tan S-B, Khin MW, Khasbazar A, Ong J, et al. SIRveNIB: selective internal radiation therapy versus sorafenib in Asia-Pacific patients with hepatocellular carcinoma. J Clin Oncol. 2018:JCO201776089. https:// doi.org/10.1200/JCO.2017.76.0892.

7. Tong AKT, Kao YH, Too C, Chin KFW, Ng DCE, Chow PKH. Yttrium-90 hepatic radioembolization: clinical review and current techniques in interventional radiology and personalized dosimetry. Br J Radiol. 2016;89:20150943.

8. Chiesa C, Sjogreen Gleisner K, Flux G, Gear J, Walrand S, Bacher K, et al. The conflict between treatment optimization and registration of radiopharmaceuticals with fixed activity posology in oncological nuclear medicine therapy. Eur J Nucl Med Mol Imaging. 2017;44:1783-6.

9. Braat AJAT, Kappadath SC, Bruijnen RCG, van den Hoven AF, Mahvash A, de Jong HWAM, et al. Adequate SIRT activity dose is as important as adequate chemotherapy dose. Lancet Oncol Elsevier Ltd. 2017;18:e636.

10. Sjögreen Gleisner K, Spezi E, Solny P, Gabina PM, Cicone F, Stokke C, et al. Variations in the practice of molecular radiotherapy and implementation of dosimetry: results from a European survey. EJNMMI Physics. 2017;4:28.

11. Giammarile F, Bodei L, Chiesa C, Flux G, Forrer F, Kraeber-Bodere F, et al. EANM procedure guideline for the treatment of liver cancer and liver metastases with intra-arterial radioactive compounds. Eur J Nucl Med Mol Imaging. 2011;38:1393-406.

12. Smits MLJ, Elschot M, Sze DY, Kao YH, Nijsen JFW, lagaru AH, et al. Radioembolization dosimetry: the road ahead. Cardiovasc Intervent Radiol. 2014;38:261-9.

13. Ahmadzadehfar $H$, Sabet A, Biermann K, Muckle M, Brockmann H, Kuhl C, et al. The significance of 99mTc-MAA SPECT/CT liver perfusion imaging in treatment planning for 90Y-microsphere selective internal radiation treatment. J Nucl Med. 2010;51:1206-12.

14. Sirtex Medical Limited. Sirtex package insert [internet]; 2017. p. 3-5. [cited 2017 Oct 31]. Available from: https:// www.sirtex.com/eu/clinicians/package-insert/

15. Elschot M, Nijsen JFW, Lam MGEH, Smits ML, Prince JF, Viergever MA, et al. 99mTc-MAA overestimates the absorbed dose to the lungs in radioembolization: a quantitative evaluation in patients treated with 166Homicrospheres. Eur J Nucl Med Mol Imaging. 2014;41:1965-75.

16. Yu N, Srinivas SM, DiFilippo FP, Shrikanthan S, Levitin A, McLennan G, et al. Lung dose calculation with SPECT/CT for 90Yittrium Radioembolization of liver Cancer. Int. J. Radiat. Oncol. Elsevier Inc. 2013;85:834-9.

17. Kao YH, Magsombol BM, Toh Y, Tay KH, Chow PK, Goh AS, et al. Personalized predictive lung dosimetry by technetium-99m macroaggregated albumin SPECT/CT for yttrium-90 radioembolization. EJNMMI Res. 2014;4:33.

18. Ho S, Lau WY, Leung TWT, Chan M, Ngar YK, Johnson PJ, et al. Partition model for estimating radiation doses from yttrium-90 microspheres in treating hepatic tumours. Eur J Nucl Med. 1996;23:947-52.

19. Gulec SA, Mesoloras G, Stabin M. Dosimetric techniques in 90 Y-microsphere therapy of liver cancer : the MIRD equations for dose calculations; 2016. p. 1209-12

20. Smits ML, Nijsen JFW, van den Bosch MAAJ, MGEH L, MAD V, Huijbregts JE, et al. Holmium-166 radioembolization for the treatment of patients with liver metastases: design of the phase I HEPAR trial. J Exp Clin Cancer Res. 2010;29:70.

21. Biocompatibles UK Ltd. Package Insert-TheraSphere ${ }^{\oplus}$ Yttrium-90 Glass Microspheres-Rev. 14 [Internet]. 2014. p. 1-21. Available from: https://www.btg-im.com/BTG/media/TheraSphere-Documents/PDF/TheraSpherePackage-Insert_USA_Rev-14.pdf.

22. Gray B, Van Hazel G, Hope M, Burton M, Moroz P, Anderson J, et al. Randomised trial of SIR-spheres ${ }^{\oplus}$ plus chemotherapy vs. chemotherapy alone for treating patients with liver metastases from primary large bowel cancer. Ann Oncol. 2001;12:1711-20.

23. Vauthey JN, Abdalla EK, Doherty DA, Gertsch P, Fenstermacher MJ, Loyer EM, et al. Body surface area and body weight predict total liver volume in western adults. Liver Transplant. 2002;8:233-40.

24. Smits ML, Nijsen JFW, van den Bosch MAAJ, Lam MGEH, Vente MAD, Mali WPTM, et al. Holmium-166 radioembolisation in patients with unresectable, chemorefractory liver metastases (HEPAR trial): a phase 1, doseescalation study. Lancet Oncol. 2012;13:1025-34.

25. Coldwell D, Sangro B, Salem R, Wasan H, Kennedy A. Radioembolization in the treatment of unresectable liver tumors: experience across a range of primary cancers. Am J Clin Oncol. 2012;35:167-77.

26. Kennedy AS, McNeillie P, Dezarn WA, Nutting C, Sangro B, Wertman D, et al. Treatment parameters and outcome in 680 treatments of internal radiation with resin $90 \mathrm{Y}$-microspheres for unresectable hepatic tumors. Int J Radiat Oncol Biol Phys. 2009;74:1494-500.

27. Braat MNGJA, van Erpecum KJ, Zonnenberg BA, van den Bosch MAJ, Lam MGEH. Radioembolization-induced liver disease. Eur J Gastroenterol Hepatol. 2017;29:144-52.

28. Sze DY, Lam MGEH. Reply to "the limitations of theoretical dose modeling for yttrium-90 radioembolization". J Vasc Interv Radiol. 2014;25:1147-8. 
29. Kao YH, Tan EH, Ng CE, Goh SW. Clinical implications of the body surface area method versus partition model dosimetry for yttrium-90 radioembolization using resin microspheres: a technical review. Ann Nucl Med. 2011;25:455-61.

30. Flux G, Bardies M, Chiesa C, Monsieurs M, Savolainen S, Strand S-E, et al. Clinical radionuclide therapy dosimetry: the quest for the "holy gray". Eur J Nucl Med Mol Imaging. 2007;34:1699-700.

31. Lam MGEH, Louie JD, Abdelmaksoud MHK, Fisher GA, Cho-Phan CD, Sze DY. Limitations of body surface areabased activity calculation for radioembolization of hepatic metastases in colorectal cancer. J Vasc Interv Radiol Elsevier. 2014;25:1085-93.

32. Bernardini M, Smadja C, Faraggi M, Orio S, Petitguillaume A, Desbrée A, et al. Liver selective internal radiation therapy with $90 \mathrm{Y}$ resin microspheres: comparison between pre-treatment activity calculation methods. Phys Medica. 2014;30:752-64.

33. Samim M, van Veenendaal LM, Braat MNGJA, van den Hoven AF, Van Hillegersberg R, Sangro B, et al. Recommendations for radioembolisation after liver surgery using yttrium-90 resin microspheres based on a survey of an international expert panel. Eur Radiol. 2017;27:4923-30.

34. Gnesin S, Canetti L, Adib S, Cherbuin N, Silva Monteiro M, Bize P, et al. Partition model-based 99mTc-MAA SPECT/ $\mathrm{CT}$ predictive dosimetry compared with 90Y TOF PET/CT posttreatment dosimetry in radioembolization of hepatocellular carcinoma: a quantitative agreement comparison. J Nucl Med. 2016;57:1672-8.

35. Wondergem M, Smits MLJ, Elschot M, de Jong HWAM, Verkooijen HM, van den Bosch MAAJ, et al. 99mTcmacroaggregated albumin poorly predicts the intrahepatic distribution of $90 \mathrm{Y}$ resin microspheres in hepatic Radioembolization. J Nucl Med. 2013;54:1294-301.

36. Ilhan H, Goritschan A, Paprottka P, Jakobs TF, Fendler WP, Todica A, et al. Predictive value of 99mTc-MAA SPECT for $90 \mathrm{Y}$-labeled resin microsphere distribution in Radioembolization of primary and secondary hepatic tumors. J Nucl Med. 2015;56:1654-60.

37. Haste P, Tann M, Persohn S, LaRoche T, Aaron V, Mauxion T, et al. Correlation of technetium-99m macroaggregated albumin and Yttrium-90 glass microsphere biodistribution in hepatocellular carcinoma: a retrospective review of pretreatment single photon emission $\mathrm{CT}$ and posttreatment positron emission tomography/CT. J Vasc Interv Radiol Elsevier Inc. 2017;28(5):722-30.

38. Gulec SA, Mesoloras G, Stabin M. Dosimetric techniques in 90Y-microsphere therapy of liver cancer: the MIRD equations for dose calculations. J Nucl Med. 2006;47:1209-11.

39. Mikell JK, Mahvash A, Siman W, Baladandayuthapani V, Mourtada F, Kappadath SC. Selective internal radiation therapy with Yttrium-90 glass microspheres: biases and uncertainties in absorbed dose calculations between clinical dosimetry models. Int J Radiat Oncol Biol Phys Elsevier Inc. 2016;96:888-96.

40. Kao YH, Hock Tan AE, Burgmans MC, Irani FG, Khoo LS, Gong Lo RH, et al. Image-guided personalized predictive dosimetry by artery-specific SPECT/CT partition modeling for safe and effective $90 \mathrm{Y}$ radioembolization. J Nucl Med. 2012:53:559-66.

41. Vogel W, van Dalen JA, Wiering B, Huisman H, Corstens FHM, Ruers TJM, et al. Evaluation of image registration in PET/CT of the liver and recommendations for optimized imaging. J Nucl Med. 2007:48:910-9.

42. Boda-Heggemann J, Knopf AC, Simeonova-Chergou A, Wertz H, Stieler F, Jahnke A, et al. Deep inspiration breath hold-based radiation therapy: a clinical review. Int J Radiat Oncol Biol Phys. 2016;94:478-92.

43. Kruis MF, van de Kamer JB, Belderbos JSA, Sonke J-J, van Herk M. 4D CT amplitude binning for the generation of a time-averaged 3D mid-position CT scan. Phys Med Biol. 2014;59:5517.

44. Liu C, Pierce IILA, Alessio AM, Kinahan PE. The impact of respiratory motion on tumor quantification and delineation in static PET/CT imaging. Phys Med Biol. 2009;54:7345-62.

45. McClelland JR, Hawkes DJ, Schaeffter T, King AP. Respiratory motion models: a review. Med Image Anal Elsevier BV. 2013;17:19-42

46. Siman W, Mawlawi OR, Mikell JK, Mourtada F, Kappadath SC. Effects of image noise, respiratory motion, and motion compensation on 3D activity quantification in count-limited PET images. Phys Med Biol IOP Publishing. 2017:62:448-64.

47. Bastiaannet R, Viergever MA, de Jong HWAM. Impact of respiratory motion and acquisition settings on SPECT liver dosimetry for radioembolization. Med Phys. 2017:44:5270-9.

48. Kessler RM, Ellis JR, Eden M. Analysis of emission tomographic scan data: limitations imposed by resolution and background. J Comput Assist Tomogr. 1984;8:514-22.

49. King MA, Long DT, Brill AB. SPECT volume quantitation: influence of spatial resolution, source size and shape, and voxel size. Med Phys. 1991;18:1016-24.

50. Lee JA. Segmentation of positron emission tomography images: some recommendations for target delineation in radiation oncology. Radiother Oncol Elsevier Ireland Ltd. 2010;96:302-7.

51. Garin E, Lenoir L, Rolland $Y$, Laffont $S$, Pracht M, Mesbah H, et al. Effectiveness of quantitative MAA SPECT/CT for the definition of vascularized hepatic volume and dosimetric approach: phantom validation and clinical preliminary results in patients with complex hepatic vascularization treated with yttrium-90-labeled micr. Nucl Med Commun. 2011:32:1245-55.

52. Garin $E$, Rolland $Y$, Lenoir L, Pracht M, Mesbah H, Porée $P$, et al. Utility of quantitative 99m Tc-MAA SPECT/CT for 90 yttrium-labelled microsphere treatment planning: calculating vascularized hepatic volume and Dosimetric approach. Int J Mol Imaging. 2011;2011:1-8.

53. Lam MGEH, Goris ML, lagaru AH, Mittra ES, Louie JD, Sze DY. Prognostic utility of 90Y radioembolization dosimetry based on fusion 99mTc-macroaggregated albumin-99mTc-sulfur colloid SPECT. J Nucl Med. 2013; 54:2055-61.

54. Kao Y-H, Steinberg JD, Tay Y-S, Lim GK, Yan J, Townsend DW, et al. Post-radioembolization yttrium-90 PET/CT part 2: dose-response and tumor predictive dosimetry for resin microspheres. EJNMMI Res. 2013;3:57.

55. Eaton BR, Kim HS, Schreibmann E, Schuster DM, Galt JR, Barron B, et al. Quantitative dosimetry for yttrium-90 radionuclide therapy: tumor dose predicts fluorodeoxyglucose positron emission tomography response in hepatic metastatic melanoma. J Vasc Interv Radiol. 2014;25:288-95. 
56. Cremonesi M, Chiesa C, Strigari L, Ferrari M, Botta F, Guerriero F, et al. Radioembolization of hepatic lesions from a radiobiology and dosimetric perspective. Front Oncol. 2014;4:1-20.

57. Flamen $P$, Vanderlinden B, Delatte $P$, Ghanem G, Ameye L, Van Den Eynde $M$, et al. Multimodality imaging can predict the metabolic response of unresectable colorectal liver metastases to radioembolization therapy with Yttrium-90 labeled resin microspheres. Phys Med Biol. 2008;53:6591-603.

58. Srinivas SM, Natarajan N, Kuroiwa J, Gallagher S, Nasr E, Shah SN, et al. Determination of radiation absorbed dose to primary liver tumors and normal liver tissue using post-Radioembolization 90Y PET. Front Oncol. 2014;4:255.

59. Willowson KP, Hayes AR, Chan DLH, Tapner M, Bernard EJ, Maher R, et al. Clinical and imaging-based prognostic factors in radioembolisation of liver metastases from colorectal cancer: a retrospective exploratory analysis. EJNMMI Res. 2017;7:46.

60. Fowler JF. 21 years of biologically effective dose. Br J Radiol. 2010;83:554-68.

61. Chiesa C, Mira M, Maccauro M, Spreafico C, Romito R, Morosi C, et al. Radioembolization of hepatocarcinoma with $90 \mathrm{Y}$ glass microspheres: development of an individualized treatment planning strategy based on dosimetry and radiobiology. Eur J Nucl Med Mol Imaging. 2015;42:1718-38.

62. Botta F, Ferrari M, Chiesa C, Vitali S, Guerriero F, De Nile MC, et al. Impact of missing attenuation and scatter corrections on 99mTc-MAA SPECT 3D dosimetry for liver radioembolization using the patient relative calibration methodology: a retrospective investigation on clinical images. Med Phys. 2018;45:1684-98.

63. Jones LC, Hoban PW. Treatment plan comparison using equivalent uniform biologically effective dose (EUBED). Phys Med Biol. 2000;45:159-70.

64. Choi H, Charnsangavej C, Faria SC, Macapinlac HA, Burgess MA, Patel SR, et al. Correlation of computed tomography and positron emission tomography in patients with metastatic gastrointestinal stromal tumor treated at a single institution with imatinib mesylate: proposal of new computed tomography response criteria. J Clin Oncol. 2007;25:1753-9.

65. Kim MN, Kim BK, Han K-H, Kim SU. Evolution from WHO to EASL and mRECIST for hepatocellular carcinoma: considerations for tumor response assessment. Expert Rev Gastroenterol Hepatol. 2015;9:335-48.

66. Riaz A, Memon K, Miller FH, Nikolaidis P, Kulik LM, Lewandowski R, et al. Role of the EASL, RECIST, and WHO response guidelines alone or in combination for hepatocellular carcinoma: radiologic-pathologic correlation. J Hepatol. 2011;54:695-704.

67. Hipps D, Ausania F, Manas DM, Rose JDG, French JJ. Selective Interarterial radiation therapy (SIRT) in colorectal liver metastases: how do we monitor response? HPB Surg. 2013;2013:570808.

68. Strigari L, Sciuto R, Rea S, Carpanese L, Pizzi G, Soriani A, et al. Efficacy and toxicity related to treatment of hepatocellular carcinoma with 90Y-SIR spheres: radiobiologic considerations. J Nucl Med. 2010;51:1377-85.

69. Chiesa C, Mira M, Maccauro M, Romito R, Spreafico C, Sposito C, et al. A dosimetric treatment planning strategy in radioembolization of hepatocarcinoma with 90Y glass microspheres. Q J Nucl Med Mol Imaging. 2012;56:503-9.

70. Fox RA, Klemp PF, Egan G, Mina LL, Burton MA, Gray BN. Dose distribution following selective internal radiation therapy. Int J Radiat Oncol Biol Phys. 1991;21:463-7.

71. Yorke ED, Jackson A, Fox RA, Wessels BW, Gray BN. Can current models explain the lack of liver complications in Y-90 microsphere therapy? Clin Cancer Res. 1999;5:3024s-30s.

72. Walrand S, Hesse M, Chiesa C, Lhommel R, Jamar F. The low hepatic toxicity per Gray of $90 \mathrm{Y}$ glass microspheres is linked to their transport in the arterial tree favoring a nonuniform trapping as observed in posttherapy PET imaging. J Nucl Med. 2014;55:135-40.

73. Högberg J, Rizell M, Hultborn R, Svensson J, Henrikson O, Mölne J, et al. Increased absorbed liver dose in selective internal radiation therapy (SIRT) correlates with increased sphere-cluster frequency and absorbed dose inhomogeneity. EJNMMI Phys. 2015;2:10.

74. Högberg J, Rizell M, Hultborn R, Svensson J, Henrikson O, Mölne J, et al. Simulation model of microsphere distribution for selective internal radiation therapy agrees with observations. Int J Radiat Oncol. 2016;96:414-21.

75. Pasciak AS, Bourgeois AC, Bradley YCA. Microdosimetric analysis of absorbed dose to tumor as a function of number of microspheres per unit volume in 90Y Radioembolization. J Nucl Med. 2016;57:1020-6.

76. Campbell AM, Bailey $\mathbb{H}$, Burton MA. Analysis of the distribution of intra-arterial microspheres in human liver following hepatic yttrium-90 microsphere therapy. Phys Med Biol. 2000;45:1023-33.

77. Dewaraja YK, Frey EC, Sgouros G, Brill AB, Roberson P, Zanzonico PB, et al. MIRD pamphlet no. 23: quantitative SPECT for patient-specific 3-dimensional dosimetry in internal radionuclide therapy. J Nucl Med. 2012;53:1310-25.

78. Pacilio M, Ferrari M, Chiesa C, Lorenzon L, Mira M, Botta F, et al. Impact of SPECT corrections on 3Ddosimetry for liver transarterial radioembolization using the patient relative calibration methodology. Med Phys. 2016;43:4053-64

79. Frey EC, Humm JL, Ljungberg M. Accuracy and precision of radioactivity quantification in nuclear medicine images. Semin Nucl Med. 2013;42:208-18.

80. Bailey DL, Willowson KP. Quantitative SPECT/CT: SPECT joins PET as a quantitative imaging modality. Eur J NuCl Med Mol Imaging. 2014;41:17-25.

81. Bailey DL, Willowson KP. An evidence-based review of quantitative SPECT imaging and potential clinical applications. J Nucl Med. 2013;54:83-9.

82. Zeintl J, Vija AH, Yahil A, Hornegger J, Kuwert T. Quantitative accuracy of clinical 99mTc SPECT/CT using orderedsubset expectation maximization with 3-dimensional resolution recovery, attenuation, and scatter correction. Nucl Med. 2010;51:921-8.

83. Vija H. Introduction to XSPECT* technology: evolving multi-modal SPECT to become context-based and quantitative [internet]. 2013

84. Elschot M, Nijsen JFW, Dam AJ, de Jong HWAM. Quantitative evaluation of scintillation camera imaging characteristics of isotopes used in liver radioembolization. PLoS One. 2011;6:e26174.

85. Shen S, DeNardo GL, Yuan A, DeNardo DA, DeNardo SJ. Planar gamma camera imaging and quantitation of yttrium-90 bremsstrahlung. J Nucl Med. 1994;35:1381-9.

86. Rong $X$, Du Y, Ljungberg M, Rault E, Vandenberghe S, Frey EC. Development and evaluation of an improved quantitative 90Y bremsstrahlung SPECT method. Med Phys. 2012;39:2346.

87. Elschot M, Lam MGEH, van den Bosch MAAJ, Viergever MA, de Jong HWAM. Quantitative Monte Carlo-based $90 \mathrm{Y}$ SPECT reconstruction. J Nucl Med. 2013;54:1557-63. 
88. Minarik D, Sjögreen Gleisner K, Ljungberg M. Evaluation of quantitative (90)Y SPECT based on experimental phantom studies. Phys Med Biol. 2008;53:5689-703.

89. Lhommel R, Goffette P, Van Den Eynde M, Jamar F, Pauwels S, Bilbao Jl, et al. Yttrium-90 TOF PET scan demonstrates high-resolution biodistribution after liver SIRT. Eur J Nucl Med Mol Imaging. 2009;36:1696.

90. Gates VL, Esmail AAH, Marshall K, Spies S, Salem R. Internal pair production of 90Y permits hepatic localization of microspheres using routine PET: proof of concept. J Nucl Med. 2011;52:72-6.

91. Carlier T, Eugène T, Bodet-Milin C, Garin E, Ansquer C, Rousseau C, et al. Assessment of acquisition protocols for routine imaging of Y-90 using PET/CT. EJNMMI Res. 2013;3:11.

92. Gates VL, Salem R, Lewandowski RJ. Positron emission tomography/CT after yttrium-90 radioembolization: current and future applications. J. Vasc. Interv. Radiol. Elsevier. 2013;24:1153-5.

93. Willowson K, Forwood N, Jakoby BW, Smith AM, Bailey DL. Quantitative 90 Y image reconstruction in PET. Med Phys. 2012;39:7153-9.

94. Wright CL, Binzel K, Zhang J, Wuthrick EJ, Knopp MV. Clinical feasibility of 90Y digital PET/CT for imaging microsphere biodistribution following radioembolization. Eur J Nucl Med Mol Imaging. 2017;44(7):1194-7.

95. Fourkal E, Veltchev I, Lin M, Koren S, Meyer J, Doss M, et al. 3D inpatient dose reconstruction from the PET-CT imaging of 90Y microspheres for metastatic cancer to the liver: feasibility study. Med Phys. 2013;40:081702.

96. Song YS, Paeng JC, Kim H-C, Chung JW, Cheon GJ, Chung J-K, et al. PET/CT-based dosimetry in 90Y-microsphere selective internal radiation therapy. Medicine (Baltimore). 2015;94:e945.

97. Ng SC, Lee VH, Law MW, Liu RK, Ma WW, Tso WK, et al. Patient dosimetry for 90Y selective internal radiation treatment based on 90Y PET imaging. J Appl Clin Med Phys. 2013;14:212-21.

98. Carlier T, Willowson KP, Fourkal E, Bailey DL, Doss M, Conti M. 90 Y -PET imaging: exploring limitations and accuracy under conditions of low counts and high random fraction. Med Phys. 2015;42:4295-309.

99. Strydhorst J, Carlier T, Dieudonne A, Conti M, Buvat I. A gate evaluation of the sources of error in quantitative $90 \mathrm{Y}$ PET. Med Phys. 2016:43:5320-9.

100. van Elmbt L, Vandenberghe S, Walrand S, Pauwels S, Jamar F. Comparison of yttrium-90 quantitative imaging by TOF and non-TOF PET in a phantom of liver selective internal radiotherapy. Phys Med Biol. 2011;56:6759-77.

101. Eldib M, Oesingmann N, Faul DD, Kostakoglu L, Knešaurek K, Fayad ZA. Optimization of yttrium-90 PET for simultaneous PET/MR imaging: a phantom study. Med Phys. 2016;43:4768-74.

102. Boellaard R, Delgado-Bolton R, Oyen WJG, Giammarile F, Tatsch K, Eschner W, et al. FDG PET/CT: EANM procedure guidelines for tumour imaging: version 2.0. Eur J Nucl Med Mol Imaging. 2014;42:328-54.

103. Willowson KP, Tapner M, The QUEST Investigator Team, Bailey DL, Willowson KP, Tapner MJ, et al. A multicentre comparison of quantitative $90 \mathrm{Y}$ PET/CT for dosimetric purposes after radioembolization with resin microspheres: the QUEST phantom study. Eur J Nucl Med Mol Imaging. 2015;42:1202-22.

104. Padia SA, Alessio A, Kwan SW, Lewis DH, Vaidya S, Minoshima S. Comparison of positron emission tomography and bremsstrahlung imaging to detect particle distribution in patients undergoing yttrium-90 radioembolization for large hepatocellular carcinomas or associated portal vein thrombosis. J Vasc Interv Radiol. 2013;24:1147-53.

105. Kao YH, Tan EH, Ng CE, Goh SW. Yttrium-90 time-of-flight PET/CT is superior to bremsstrahlung SPECT/CT for postradioembolization imaging of microsphere biodistribution. Clin Nucl Med. 2011;36:e186-7.

106. Elschot M, Vermolen BJ, Lam MGEH, de Keizer B, van den Bosch MAAJ, de Jong HWAM. Quantitative comparison of PET and bremsstrahlung SPECT for imaging the in vivo Yttrium-90 microsphere distribution after liver Radioembolization. PLoS One. 2013;8:e55742.

107. Seevinck PR, Seppenwoolde J-H, de Wit TC, Nijsen JFW, Beekman FJ, van Het Schip AD, et al. Factors affecting the sensitivity and detection limits of MRI, CT, and SPECT for multimodal diagnostic and therapeutic agents. Anti Cancer Agents Med Chem. 2007;7:317-34.

108. Van De Maat GH, Seevinck PR, Elschot M, Smits MLJ, De Leeuw H, Van Het Schip AD, et al. MRI-based biodistribution assessment of holmium-166 poly(L-lactic acid) microspheres after radioembolisation. Eur Radiol. 2013;23:827-35.

109. Elschot M, Smits ML, Nijsen JFW, Lam MGEH, Zonnenberg BA, van den Bosch MAAJ, et al. Quantitative Monte Carlo-based holmium-166 SPECT reconstruction. Med Phys. 2013:40:112502.

110. de Wit TC, Xiao J, JFW N, van het Schip FD, Staelens SG, van Rijk PP, et al. Hybrid scatter correction applied to quantitative holmium-166 SPECT. Phys Med Biol. 2006;51:4773-87.

111. Smits ML, Elschot M, Van Den Bosch MAAJ, Van De Maat GH, Van Het Schip AD, Zonnenberg BA, et al. In vivo dosimetry based on SPECT and MR imaging of 166 ho-microspheres for treatment of liver malignancies. J Nucl Med. 2013;54:2093-100.

112. Kawrakow I. The EGSnrc code system: Monte Carlo simulation of Electron and photon transport. Med Phys. 2007;34:4818-53.

113. Goorley JT, James MR, Booth TE, Brown FB, Bull JS, Cox LJ, et al. Initial MCNP6 Release Overview - MCNP6 version 1.0. Los Alamos National Lab. 2013. https://doi.org/10.2172/1086758. Accessed 26 June 2018.

114. Andersen V, Ballarini F, Battistoni G, Cerutti F, Empl A, Fassò A, et al. The application of FLUKA to dosimetry and radiation therapy. Radiat Prot Dosim. 2005;116:113-7.

115. Sarrut D, Bardiès M, Boussion N, Freud N, Jan S, Létang J-M, et al. A review of the use and potential of the GATE Monte Carlo simulation code for radiation therapy and dosimetry applications. Med Phys. 2014;41:064301.

116. Dieudonne A, Hobbs RF, Lebtahi R, Maurel F, Baechler S, Wahl RL, et al. Study of the impact of tissue density heterogeneities on 3-dimensional abdominal dosimetry: comparison between dose kernel convolution and direct Monte Carlo methods. J Nucl Med. 2013;54:236-43.

117. Mikell JK, Mahvash A, Siman W, Mourtada F, Kappadath SC. Comparing voxel-based absorbed dosimetry methods in tumors, liver, lung, and at the liver-lung interface for $90 \mathrm{Y}$ microsphere selective internal radiation therapy. EJNMMI Phys. 2015;2:16.

118. Pasciak AS, Erwin WD. Effect of voxel size and computation method on Tc-99m MAA SPECT/CT-based dose estimation for Y-90 microsphere therapy. IEEE Trans Med Imaging. 2009;28:1754-8.

119. Pasciak AS, Bourgeois AC, Bradley YC. A comparison of techniques for (90)Y PET/CT image-based dosimetry following Radioembolization with resin microspheres. Front Oncol. 2014;4:121. 
120. Bourgeois AC, Chang TT, Bradley YC, Acuff SN, Pasciak AS. Intraprocedural yttrium-90 positron emission tomography/CT for treatment optimization of yttrium-90 radioembolization. J. Vasc. Interv. Radiol. Elsevier. 2014; 25:271-5.

121. Walrand S, Hesse M, Demonceau G, Pauwels S, Jamar F. Yttrium-90-labeled microsphere tracking during liver selective internal radiotherapy by bremsstrahlung pinhole SPECT: feasibility study and evaluation in an abdominal phantom. EJNMMI Res. 2011;1:32.

122. Beijst C, Elschot M, Viergever MA, de Jong HWAM. Toward simultaneous real-time fluoroscopic and nuclear imaging in the intervention room. Radiology. 2016;278:232-8.

123. Van Der Velden S, Beijst C, Viergever MA, De Jong HWAM. Simultaneous fluoroscopic and nuclear imaging: impact of collimator choice on nuclear image quality: impact. Med Phys. 2017:44:249-61.

124. van der Velden S, Bastiaannet R, Braat AJAT, Lam MGEH, Viergever MA, de Jong HWAM. Estimation of lung shunt fraction from simultaneous fluoroscopic and nuclear images. Phys Med Biol. 2017;62:8210-25.

125. Seppenwoolde JH, Bartels LW, Van Der Weide R, Nijsen JFW, Van Het Schip AD, Bakker CJG. Fully MR-guided hepatic artery catheterization for selective drug delivery: a feasibility study in pigs. J Magn Reson Imaging. 2006; 23:123-9.

126. Ritt P, Vija H, Hornegger J, Kuwert T. Absolute quantification in SPECT. Eur J Nucl Med Mol Imaging. 2011;38:69-77.

127. Büther F, Vehren T, Schäfers KP, Schäfers M. Impact of data-driven respiratory gating in clinical PET. Radiology. 2016;281:229-38

128. Kesner AL, Chung JH, Lind KE, Kwak JJ, Lynch D, Burckhardt D, et al. Validation of software gating: a practical Technology for Respiratory Motion Correction in PET. Radiology. 2016;281:239-48.

129. Bastiaannet $R$, Van Der Velden S, Lam M, Viergever M, de Jong H. Fast quantitative determination of lung shunt fraction using orthogonal planar projections in hepatic radioembolization. J Nucl Med Soc Nuclear Med. 2016;57:537.

130. Eberlein U, Cremonesi M, Lassmann M. Individualized dosimetry for Theranostics: necessary, nice to have, or counterproductive? J Nucl Med. 2017;58:97S-103S.

131. Selwyn RG, Avila-Rodriguez MA, Converse AK, Hampel JA, Jaskowiak CJ, McDermott JC, et al. 18F-labeled resin microspheres as surrogates for $90 \mathrm{Y}$ resin microspheres used in the treatment of hepatic tumors: a radiolabeling and PET validation study. Phys Med Biol. 2007;52:7397-408.

132. Schiller E, Bergmann R, Pietzsch J, Noll B, Sterger A, Johannsen B, et al. Yttrium-86-labelled human serum albumin microspheres: relation of surface structure with in vivo stability. Nucl Med Biol. 2008;35:227-32.

133. Grosser O, Ruf J, Kupitz D, Pethe A, Ulrich G, Genseke P, et al. Pharmacokinetics of 99mTc-MAA- and 99mTc-HSAMicrospheres Used in Preradioembolization Dosimetry: Influence on the Liver-Lung Shunt. J Nucl Med. 2016;57(6): 925-7.

134. Kappadath SC, Mikell J, Balagopal A, Baladandayuthapani V, Kaseb A, Mahvash A. Hepatocellular carcinoma tumor dose response following 90 Y-radioembolization with glass microspheres using 90 Y-SPECT/CT based voxel dosimetry. Int. J. Radiat. Oncol. Elsevier Inc. 2018. https://doi.org/10.1016/j.jjrobp.2018.05.062.

135. Garin E, Lenoir L, Edeline J, Laffont S, Mesbah H, Porée $P$, et al. Boosted selective internal radiation therapy with $90 \mathrm{Y}$-loaded glass microspheres (B-SIRT) for hepatocellular carcinoma patients: a new personalized promising concept. Eur J Nucl Med Mol Imaging. 2013;40:1057-68.

136. Garin E, Lenoir L, Rolland Y, Edeline J, Mesbah H, Laffont S, et al. Dosimetry based on 99mTc-macroaggregated albumin SPECT/CT accurately predicts tumor response and survival in hepatocellular carcinoma patients treated with 90Y-loaded glass microspheres: preliminary results. J Nucl Med. 2012:53:255-63.

137. Chan KT, Alessio AM, Johnson GE, Vaidya S, Kwan SW, Monsky W, et al. Prospective trial using internal pairproduction positron emission tomography to establish the Yttrium-90 radioembolization dose required for response of hepatocellular carcinoma. Int J Radiat Oncol Elsevier Inc. 2018;101:358-65.

138. Fowler KJ, Maughan NM, Laforest R, Saad NE, Sharma A, Olsen J, et al. PET/MRI of hepatic 90Y microsphere deposition determines individual tumor response. Cardiovasc Intervent Radiol. 2016;39:855-64.

139. Flamen $P$, Vanderlinden $B$, Delatte $P$, Ghanem $G$, Ameye L, Van Den Eynde $M$, et al. Corrigendum: multimodality imaging can predict the metabolic response of unresectable colorectal liver metastases to radioembolization therapy with Yttrium-90 labeled resin microspheres (2008 Phys. Med. Biol. 53 6591-603). Phys Med Biol. 2014;59: 2549-51.

140. van den Hoven AF, Rosenbaum CENM, Elias SG, de Jong HWAM, Koopman M, Verkooijen HM, et al. Insights into the dose-response relationship of Radioembolization with resin 90Y-microspheres: a prospective cohort study in patients with colorectal Cancer liver metastases. J Nucl Med. 2016;57:1014-9.

141. Chansanti O, Jahangiri Y, Matsui Y, Adachi A, Geeratikun Y, Kaufman JA, et al. Tumor dose response in Yttrium-90 resin microsphere embolization for neuroendocrine liver metastases: a tumor-specific analysis with dose estimation using SPECT-CT. J Vasc Interv Radiol SIR. 2017;28(11):1528-35. 